\title{
Hochwasser und Sturzfluten an Flüssen in Deutschland
}

Axel Bronstert, Helge Bormann, Gerd Bürger, Uwe Haberlandt, Fred Hattermann, Maik Heistermann, Shaochun Huang, Vassilis Kolokotronis, Zbigniew Kundzewicz, Lucas Menzel, Günter Meon, Bruno Merz, Andreas Meuser, Eva Nora Paton, Theresia Petrow

10.1 Hochwasser in Flussgebieten der Mesoskala - 88

10.1.1 Ergebnisse für Deutschland insgesamt - 88

10.1.2 Ergebnisse für Flussgebiete in Südwest- und Süddeutschland - 91

10.1.3 Ergebnisse für den Rhein - 92

10.1.4 Ergebnisse für das obere Elbegebiet - 94

10.1.5 Ergebnisse für das Weser- und Emsgebiet - 96

10.2 Sturzfluten und Extremniederschläge kurzer Dauer - 97

10.2.1 Spezifika von Sturzfluten - 97

10.2.2 Datenanalyse zur Entwicklung von hochintensiven Starkregenereignissen - 98

10.2.3 Zur künftigen Entwicklung von hoch intensiven Starkregenereignissen - 99

10.3 Kurz gesagt -99

Literatur - 100 
Durch Starkniederschläge ausgelöste Flusshochwasser sind in Deutschland die Naturereignisse, die die größten wirtschaftlichen Schäden verursachen. Neben der niederschlagsbedingten Abflussbildung wirken häufig weitere Mechanismen, die zu lokalen Überschwemmungen führen und die in diesem Bericht nicht behandelt werden können, so etwa der Verschluss von Fließgewässerquerschnitten durch Treibgut an Brücken und Durchlässen, Rückstau an hydraulischen Engstellen oder Abflusshindernisse durch Hangrutschungen oder Eisblockaden. Ein besonderes Risiko ergibt sich aus dem Versagen von Hochwasserschutzanlagen wie z. B. Deichen.

Die Frage des möglichen Einflusses der Klimaänderungen bzw. der globalen Erwärmung auf die Hochwasserverhältnisse in Deutschland wird von der Öffentlichkeit sowie der Fachwelt intensiv und kontrovers geführt, vor allem während und kurz nach starken Hochwasserereignissen. Auch für solche Diskussionen ist eine Zusammenschau des Wissens für Deutschland umso mehr von hoher Relevanz, als in globalen Assessment Reports wenig Konkretes zur Situation in Deutschland vorhanden ist. Im Fünften Sachstandsbericht (AR5) des Weltklimarats (IPCC) ist im zweiten Kapitel der 1. Arbeitsgruppe im Unterkapitel 2.6.2.2 (Hartmann et al. 2013) zu Hochwasser lediglich zu finden:

I „ „... Trends regionaler Hochwasser sind stark von Wassermanagementmaßnahmen beeinflusst ..." und „... andere Studien in Europa und Asien zeigen Belege für steigende, fallende oder gar keine Trends ...."

Im dritten Kapitel der 2. Arbeitsgruppe ist im Unterkapitel 3.2.7 (Jiménez Cisneros 2014) zu extremen hydrologischen Ereignissen und deren Wirkungen noch erwähnt:

I) „Es gibt keine starken Belege für eine Zunahme der Hochwasser in den USA, Europa, Südamerika und Afrika. Allerdings ist in kleineren Raumskalen in Teilen von Nordwesteuropa eine Zunahme des maximalen Abflusses beobachtet worden, wogegen in Südfrankreich eine Abnahme beobachtet wurde."

Daraus wird klar, dass die Aussagen im AR5 zu Flusshochwasser im Allgemeinen und Deutschland im Besonderen sehr spärlich und für etwaige Management- oder Anpassungsmaßnahmen in Deutschland irrelevant sind.

Bei der Kategorisierung von Flusshochwasserereignissen ist es sinnvoll, nach Entstehungs- und Wirkungsmechanismen zu unterscheiden. Demnach sind Sturzfluten plötzlich eintretende Hochwasserereignisse, die durch kleinräumige Regenereignisse kurzer Dauer, aber hoher Intensität ausgelöst werden. Sie haben insbesondere für kleinere Einzugsgebiete mit kurzen Reaktionszeiten (Zeit zwischen dem auslösenden Niederschlags- und dem Hochwasserereignis) ein hohes Schadenspotenzial. Entsprechend der Dauer dieser Niederschlagsereignisse ergeben sich Sturzfluten meist im Zusammenhang mit Reaktionszeiten von weniger als 6 Stunden (Borga et al. 2011). In großen Flussgebieten werden Hochwasser dagegen durch lang anhaltende, großräumige Regenereignisse ausgelöst. Weitere Differenzierungsmerkmale liefern die verschiedenen Entstehungsmechanismen, z. B. zwischen Winter- und Sommerhochwasserereignissen, Hochwasser aufgrund von Schneeschmelze,
Hochwasser als Folge von Regen auf gesättigte Böden oder als Folge von Starkniederschlag auf wenig durchlässige Böden.

Bei der Untersuchung der Klimaänderungswirkungen auf die Hochwasser wird die Komplexität der Hochwasserentstehung häufig missachtet, was zu falschen Kausalitätsannahmen oder Fehlinterpretationen führen kann. Eine vollumfassende, d. h. flächendeckende, regionsspezifische und ereignisdifferenzierende Beurteilung möglicher Klimaänderungseffekte auf das Hochwasserregime erfordert Aussagen zu Veränderungen der Größe (sowohl nach Abflusshöhe als auch nach räumlicher Ausdehnung), der Dauer des jahreszeitlichen Auftretens und der Häufigkeit der Hochwasserereignisse in der adäquaten Raum- und Zeitskala (s. u.). Infolge der Prozess- und Systemvielfalt sind hierzu regional differenzierte Aussagen unter Berücksichtigung der maßgebenden Hochwasserentstehungsbedingungen notwendig, die hohe Anforderungen an Aussagen zu Veränderungen der meteorologischen Ursachen der Hochwasserentstehung sowie klimatischer Randbedingungen (z. B. der Vorfeuchte) stellen. Die aktuell verfügbaren pragmatischen Ansätze der Datenanalyse von Hochwasserzeitreihen und/oder die prozessbasierte Modellierung in gekoppelten meteorologisch-hydrologisch-hydraulischen Modellsystemen sind die für diese Problemstellung adäquaten Werkzeuge. Gleichwohl sind deren Ergebnisse infolge begrenzter Datenverfügbarkeit und einer modellbedingten Vernachlässigung der Komplexität meist nur von eingeschränkter Aussagefähigkeit.

Es ist zu beachten, dass zur Hochwasseranalyse adäquate Skalen zugrunde gelegt werden, d.h. Skalen, in denen die Prozesse der Abflussentstehung und -konzentration auftreten und zudem Managementmaßnahmen wirken können. Diese typische Raumskala ist die obere Mesoskala von etwa 1000 bis $100.000 \mathrm{~km}^{2}$ - also nicht kontinental oder gar global - für Hochwasser an den größeren Flüssen. Für Sturzfluten ist die adäquate Raumskala die untere Mesoskala von etwa 50 bis $1000 \mathrm{~km}^{2}$. Die relevante Zeitskala der Hochwasserentstehung liegt für große Flusshochwasser meist bei mehreren Tagen bis Wochen, bei einer zeitlichen Auflösung von Tagen. Für Sturzfluten ist die relevante Zeitskala zwischen Stunden und ca. 1 Tag, bei einer stündlichen bis ca. 5-minütlichen zeitlichen Auflösung. Letztlich gilt generell, dass Aussagen zu den Wirkungen von Umweltänderungen - z. B. zur Änderung der Landnutzung, des Klimas oder auch des Flussbaus - auf Hochwasserverhältnisse umso unschärfer werden, je seltener ein derartiges Ereignis auftritt. Mit geringerer Auftretenswahrscheinlichkeit des Ereignisses sinkt also die Zuverlässigkeit der Aussagen.

\subsection{Hochwasser in Flussgebieten der Mesoskala}

\subsubsection{Ergebnisse für Deutschland insgesamt}

\section{- (Daten-)Analyse der Vergangenheit (bis heute)}

Untersucht man langjährige Veränderungen in den hydrologischen Prozessen einer Region oder eines Einzugsgebiets, wird normalerweise unterschieden zwischen der Detektion eines Trends durch Verfahren der statistischen Zeitreihenanalyse und der Attribution des Trends, also der Zuschreibung der Ursachen (Merz et al. 2012). Schwierig ist es, wenn mehrere Einflussgrößen 


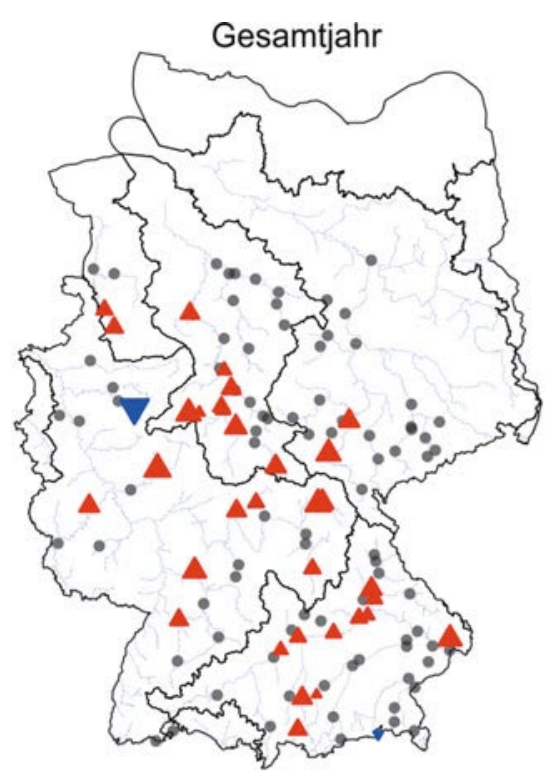

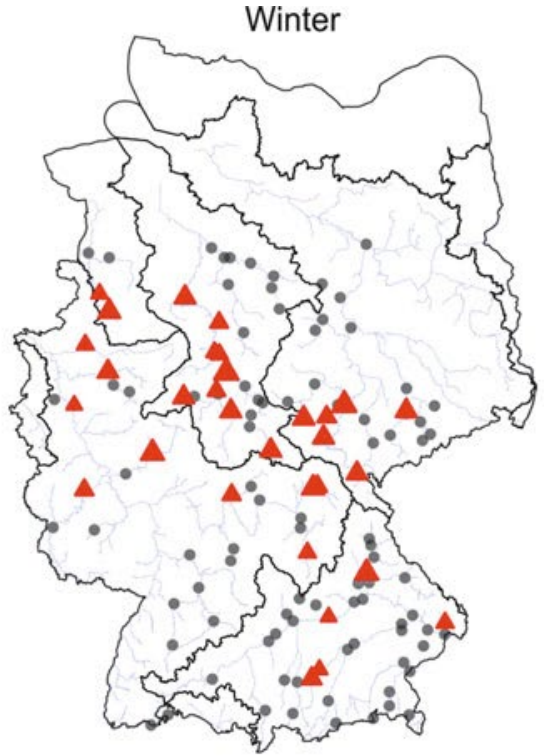

b

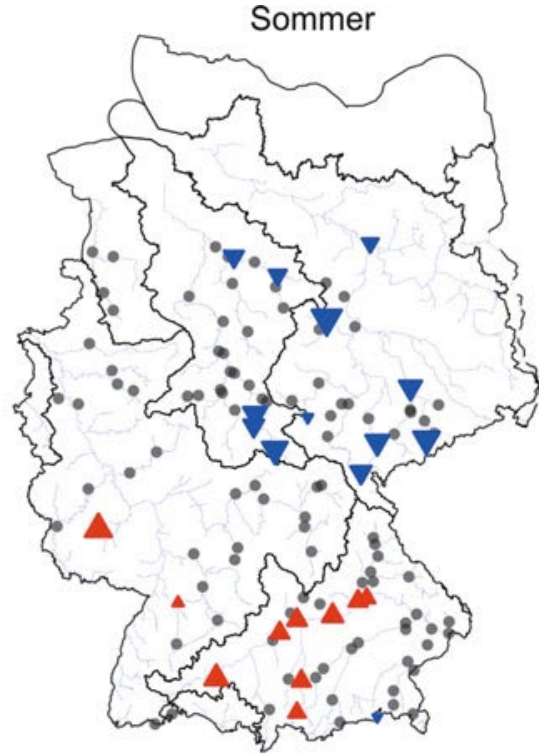

C

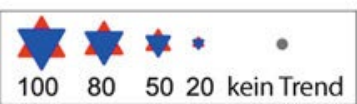

- Abb. 10.1 Räumliche Verteilung von signifikanten Trends in Jahreshöchstabflüssen. a Gesamtjahr, b Winter (November-März), c Sommer (April-Oktober). Dreiecke signifikante Trends, Graue Punkte keine signifikanten Veränderungen, Größe der Dreiecke Stärke des Trends, Blau abnehmender Trend, Rot ansteigender Trend. (Petrow und Merz 2009, geändert)

als Ursache für einen beobachteten Trend infrage kommen, wie es beim Hochwasser der Fall ist. Neben dem Klima als wichtiger Einflussgröße können auch Änderungen in der Landschaft, die in den letzten 100 Jahren besonders intensiv waren, ursächlich für Trends im Hochwassergeschehen sein - z. B. Flussbegradigungen, Versiegelung und Landschaftswandel. Zusätzlich werden Aussagen zu Veränderungen des Hochwassergeschehens dadurch erschwert, dass in der Regel nur ein Hochwassermerkmal betrachtet wird. Bei diesem Merkmal handelt es sich um den Hochwasserscheitel, der sowohl ereignisabhängig als auch infolge seiner Sensitivität gegenüber anthropogenen Einflussfaktoren sehr stark schwankt. Statistisch spricht man von einem ungünstigen TrendRausch-Verhältnis; dies bedingt eine hohe Unsicherheit bereits bei der Detektion eines Trends. Außerdem treten große Hochwasser oft gehäuft auf. Je nachdem, ob eine derartige Häufung am Beginn oder Ende des analysierten Zeitraums auftritt, ergibt sich ein (durchaus statistisch signifikanter) fallender oder steigender Trend. Hochwassertrendanalysen sind deshalb vorsichtig zu interpretieren (s. a. grundsätzliche Diskussion in Merz et al. 2012).

Petrow und Merz (2009) analysierten die Hochwassertrends an 145 Abflusspegeln für Einzugsbiete über $500 \mathrm{~km}^{2}$ Fläche, die über ganz Deutschland verteilt waren. Sie ermittelten für diese Pegel acht Hochwasserindikatoren: jährliche und saisonale Höchstabflüsse (jeweils ein Wert pro Jahr) sowie Hochwasserscheitelabflüsse, die vorgegebene Schwellenwerte überschritten, wobei auch die jährlichen Häufigkeiten dieser Überschreitungen sowohl für das Winter- als auch das Sommerhalbjahr betrachtet wurden. Diese Analysen wurden für alle Pegel für den identischen Zeitraum 1951-2002 durchgeführt.

Die Ergebnisse dieser Untersuchung des Zeitraums 19512002 lassen sich wie folgt zusammenfassen:
- Die jährlichen Maxima der Tagesabflussmittelwerte zeigten an $28 \%$ der Pegel signifikant zunehmende Trends, an nur zwei Pegeln waren fallende Trends zu beobachten. $23 \%$ der Pegel zeigten einen steigenden Trend der Wintermaxima. Die Sommermaxima wiesen an jeweils $10 \%$ der Pegel steigende bzw. fallende Trends auf. Bei der Interpretation dieser Prozentanteile muss beachtet werden, dass Hochwasserzeitreihen an benachbarten Pegeln häufig korreliert sind und somit per se ein ähnliches Trendverhalten aufweisen.

- Für die verschiedenen Hochwasserindikatoren und Flusseinzugsgebiete ergaben sich erhebliche Unterschiede. Die Einzugsgebiete der Donau und des Rheins zeigten die meisten Trends, Weser und Elbe deutlich weniger. So wies etwa ein Drittel der Pegel im westlichen und südwestlichen Teil Deutschlands signifikant steigende Trends der jährlichen Höchstabflüsse auf, wogegen fast keine steigenden Trends in Ostdeutschland (Elbe) zu verzeichnen waren.

- Für die Mehrheit aller Pegel (zwischen 71 und 97\%) wurden keine signifikanten Trends detektiert. Wenn signifikante Änderungen gefunden wurden, waren diese fast durchweg positiv, d.h., in diesen Fällen nahmen die Hochwasserscheitel bzw. -häufigkeiten zu.

- Interessant waren räumliche Clusterungen sowie saisonale Differenzierungen von Trends: z. B. im Winter ausschließlich steigende Trends, im Sommer steigende und fallende Trends (- Abb. 10.1). Trends der Wintermaxima wurden insbesondere für Pegel in Mitteldeutschland gefunden. Die Sommerhochwasser zeigten in Süddeutschland einen zunehmenden, in Ostdeutschland einen abnehmenden Trend.

- Die räumliche und saisonale Konsistenz von Trends lässt auf großräumige und saisonal unterschiedliche Ursachen 


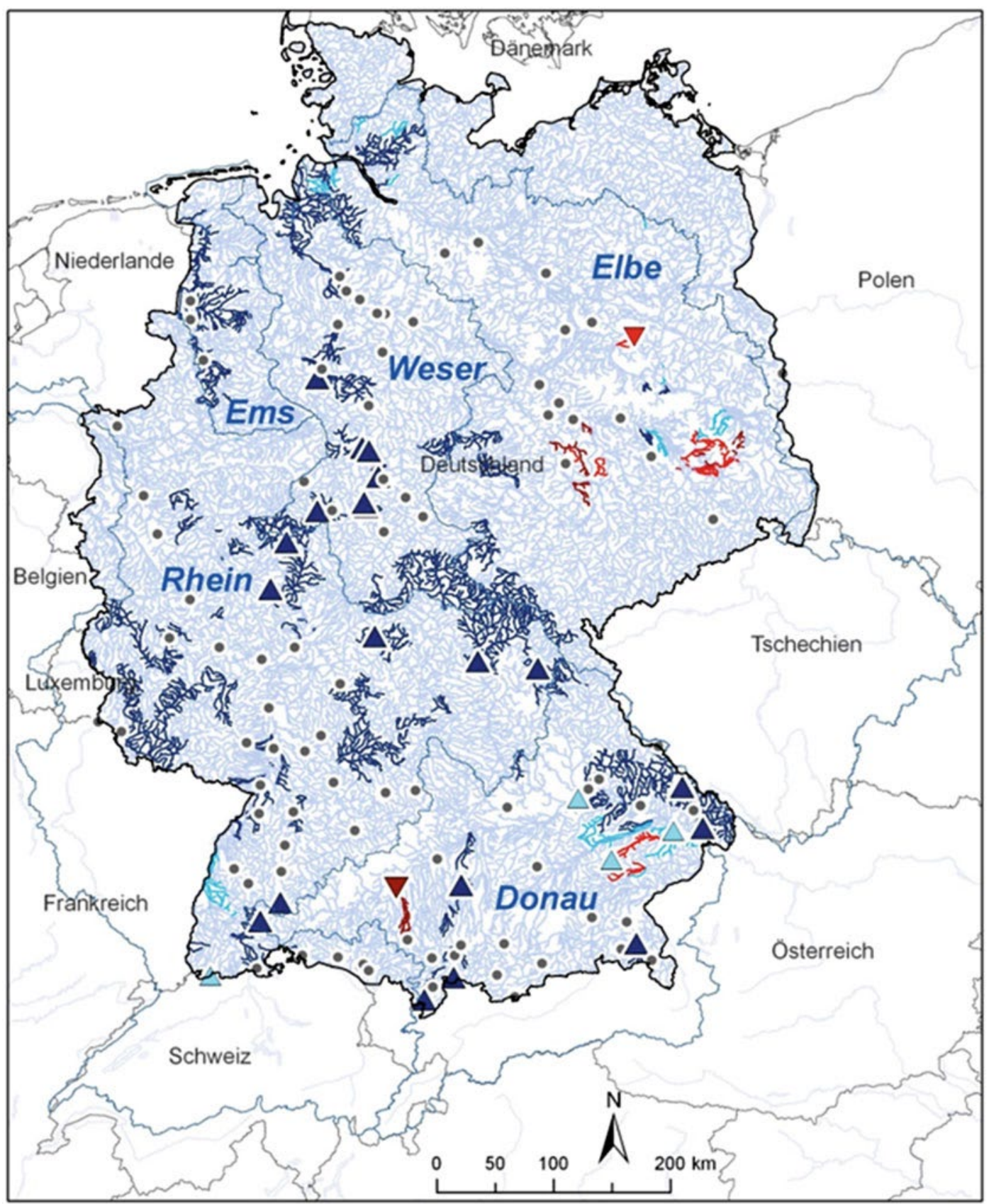

\section{Beobachtet}

$\triangle$ positiv

negativ

\section{Simuliert}

$\wedge$ positiv

$\wedge$ negativ

- Abb. 10.2 Beobachtete und simulierte Hochwassertrends für 1951-2003. (Hattermann et al. 2012, geändert)

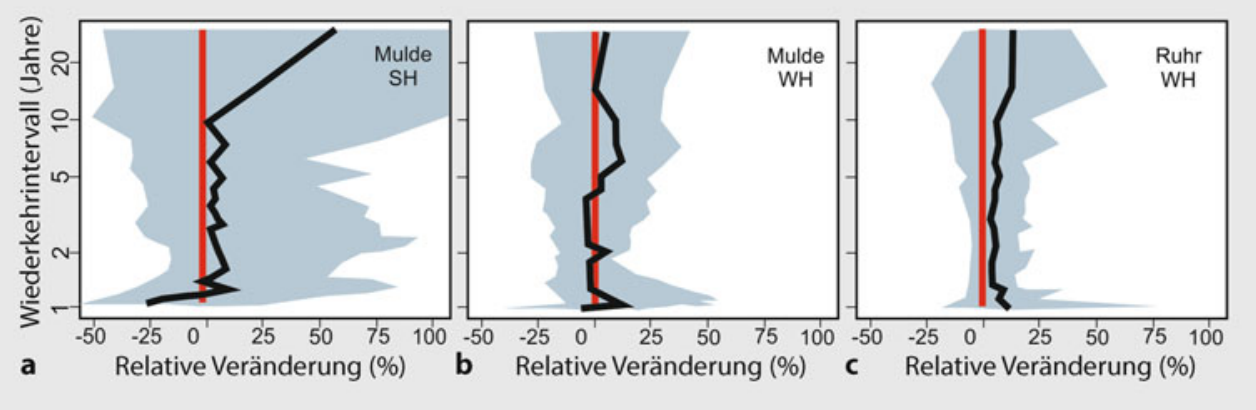

- Abb. 10.3 Simulierte Änderungen der Hochwasserabflüsse in zwei deutschen Flussgebieten (a Mulde - Sommerhalbjahr, b Mulde - Winterhalbjahr, c Ruhr - Winterhalbjahr). Gezeigt werden die relativen Unterschiede der Perioden 2021-2050 und 1971-2000, für Wiederkehrintervalle zwischen 1 und 50 Jahren. Die grau hinterlegten Bereiche markieren die Bandbreite für die Ensembleläufe. (Ott et al. 2013, geändert) 
schließen. Daher vermuten Petrow und Merz (2009) die Klimavariabilität und/oder den Klimawandel als Ursache.

Diese Studie belegt, dass sich zwischen 1951 und 2002 die Hochwasserverhältnisse in einigen Einzugsgebieten in Deutschland verändert haben. Eine zeitliche Extrapolation dieser Trends ist trotz des Auftretens großer Hochwasser in den Jahren 2005, 2006 und 2013 in den Flussgebieten von Elbe und Donau nicht zulässig, da diese Veränderungen Teil von langfristigen zyklischen Schwankungen des Hochwasserregimes sein können (Schmocker-Fackel und Naef 2010).

\section{- Attribution von Veränderungen des Hochwasserregimes über die Entwicklung der Großwetterlagen}

Insbesondere für große Flüsse besteht ein statistischer Zusammenhang zwischen den Häufigkeiten der Hochwasserereignisse und der Häufigkeit von Großwetterlagen. In Petrow et al. (2009) wird ein Zusammenhang zwischen den oben beschriebenen Trendänderungen und den täglichen Großwetterlagen über Europa (nach Hess und Brezowsky, einer subjektiven Wetterlagenklassifizierung) untersucht. Dazu wurde Deutschland in drei Regionen mit homogenem Hochwasserregime zusammengefasst. Die potenziell hochwasserauslösenden Großwetterlagen (GWL) wurden für jede Region ermittelt und anschließend die Trends in Hochwasserindikatoren für jede Region mit Trends in Häufigkeit und Persistenz von GWL verglichen. Es lässt sich ein statistisch signifikanter Trend hin zu einer geringeren Vielfalt von GWL beobachten, dafür aber eine längere Dauer. Dies gilt auch insbesondere für hochwasserauslösende GWL (Petrow et al. 2009). Dieser Anstieg von Frequenz und Andauer hochwasserträchtiger GWL kann als Ursache für den genannten Trend der zunehmenden Häufigkeit von Hochwasserereignissen im Winterhalbjahr in Deutschland interpretiert werden.

Diese Trendanalysen der GWL stützen die Hypothese, dass die Zunahme des (häufigen, also nicht extremen) Hochwasserauftretens klimatisch bedingt ist. Allerdings muss beachtet werden, dass Hochwasserzeitreihen längerfristige Fluktuationen zeigen, sodass die Ergebnisse von Trendanalysen vom betrachteten Zeitraum abhängen. Hattermann et al. (2013) verglichen für denselben Zeitraum die Regionen, in denen die Hochwasser signifikant ansteigen, mit Trends in der jährlichen Häufigkeit von Tagen mit starken Niederschlägen (von mehr als $30 \mathrm{~mm}$ pro Tag) und zeigten, dass es hier eine deutliche regionale Übereinstimmung gab.

Eine weitere Möglichkeit, die beobachteten Ursachen einer Umweltänderung kausal zuzuordnen, ist die Anwendung von prozessbasierten Modellen, welche die relevanten hydrologischen Prozesse im Modellkonzept integrieren. So betrieben Hattermann et al. (2013) für ganz Deutschland ein hydrologisches Modell (SWIM, Soil and Water Integrated Model, Krysanova et al. 1998) mit täglicher Auflösung für 1951-2003. Dabei hielten sie die Landnutzung und die wasserwirtschaftlichen Einflüsse konstant und belegten durch die hohe Übereinstimmung zwischen beobachteten und simulierten Abflüssen ( $\mathrm{Abb}$. 10.2), dass die Ursachen der durch Petrow und Merz (2009) ermittelten Trends in den jährlichen Hochwasserabflüssen für 1951-2002 nicht in der Wasserbewirtschaftung und dem Landschaftswandel, sondern eher in Änderungen der meteorologischen Eingangsgrößen liegen.
Auch Hundecha und Merz (2012) untersuchten mit einer Modellierungsstudie acht deutsche Einzugsgebiete mit unterschiedlichen Hochwasserregimen für den Zeitraum 1951-2003. Mit einem Wettergenerator wurden sowohl stationäre als auch instationäre meteorologische Felder für Niederschlag und Temperatur erzeugt. Damit wurde das hydrologische Modell SWIM angetrieben, ohne Veränderungen in den Landnutzungs- oder anderen Modellparametern. Das Ergebnis: Wo die simulierten mit den beobachteten Hochwassertrends übereinstimmen, waren diese durch Veränderungen im Niederschlag bedingt. Temperaturänderungen waren dagegen untergeordnet. Allerdings konnten die beobachteten Hochwassertrends nicht in allen Fällen durch Klimaeinflüsse erklärt werden. Dann spielten vermutlich andere Ursachen eine wesentliche Rolle, etwa Änderungen in der Landnutzung oder im Flussbau.

\section{- Modellierungsergebnisse zu künftigen Klimabedingungen}

Ott et al. (2013) untersuchten den möglichen Einfluss des künftigen Klimawandels auf Hochwasser für den Zeitraum 20212050 in drei mesoskaligen Einzugsgebieten mit verschiedenen Hochwasserregimen: Ammer, Mulde und Ruhr. Als Basisklimaszenario wurde das SRES-Szenario A1B gewählt. Davon wurde ein (kleines) klimatologisch-hydrologisches Ensemble von zehn regionalen Simulationen abgeleitet, bestehend aus der Kombination zweier hydrologischer Modelle (WaSim und SWIM) mit zwei hochaufgelösten regionalen Klimamodellen (WRF und CLM) und den Ergebnissen von zwei globalen Klimamodellen mit insgesamt vier Realisationen - drei Realisationen mit ECHAM5 (E5R1 bis E5R3) und eine Realisation vom kanadischen Modell CCCma3 (C3). Die Ergebnisse (- Abb. 10.3) zeigen, dass die durch das Ensemble abgebildete Unsicherheit groß ist und mit der Saison und dem Einzugsgebiet variiert.

\subsubsection{Ergebnisse für Flussgebiete in Südwest- und Süddeutschland}

- (Daten-)Analyse der Vergangenheit (bis heute)

Im Kooperationsvorhaben KLIWA stehen die Ermittlung bisheriger Veränderungen des Klimas und des Wasserhaushalts sowie die Abschätzung der Auswirkungen möglicher zukünftiger Klimaveränderungen auf den Wasserhaushalt für Flüsse und Einzugsgebiete in Südwest- und Süddeutschland der Bundesländer Baden-Württemberg, Bayern und Rheinland-Pfalz im Vordergrund. Für die Analyse des Langzeitverhaltens der Hochwasserkennwerte dienten Zeitreihen der Monatshöchstwerte HQ(m) der Jahre 1932-2010 von insgesamt 115 Pegeln an allen relevanten Flüssen in dieser Region (KLIWA 2011).

Für die Analyse des Langzeitverhaltens der jährlichen und halbjährlichen Abflusshöchstwerte eines Pegels wurden die monatlichen Höchstwerte des Abflusses zu Jahresserien für das hydrologische Jahr, das Sommer- und das Winterhalbjahr zusammengefasst. Für diese Serien wurde anschließend die langjährige Veränderung in Form von linearen Trends und deren statistische Signifikanzen ermittelt. Die Ergebnisse der Trenduntersuchun- 
- Tab. 10.1 Überblick über das Trendverhalten der Hochwasserabflüsse an den 115 untersuchten Pegeln in Baden-Württemberg, Bayern und Rheinland-Pfalz im Zeitraum 1932-2010

\section{Tendenzen}

Hydrologisches Gesamtjahr (November-Oktober)

$\Downarrow$ Pegel mit abnehmendem Trend/signifikant

$\diamond$ Pegel mit zunehmendem Trend/signifikant

Hydrologisches Winterhalbjahr (November-April)

$\S$ Pegel mit abnehmendem Trend/signifikant

থ Pegel mit zunehmendem Trend/signifikant

Hydrologisches Sommerhalbjahr (Mai-Oktober)

$₫$ Pegel mit abnehmendem Trend/signifikant

৯ Pegel mit zunehmendem Trend/signifikant

\section{Anzahl der Pegel mit Trend ${ }^{*}$}

$31 / 6$

$84 / 28$

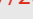

$73 / 33$

$28 / 6$

$24 / 21$

$87 / 36$

$75 / 41$

$47 / 6$

$41 / 13$

$68 / 32$

$59 / 47$

* Gesamtzahl Pegel mit vorliegendem Trend/Anzahl mit signifikantem Trend $a \geq 80 \%$ bzw. Irrtumswahrscheinlichkeit $<20 \%$

gen sind für alle 115 Pegel in $\bullet$ Tab. 10.1 zusammengefasst. Die Analyse für 1932-2010 zeigt für 84 Pegel bezogen auf das Gesamtjahr einen ansteigenden Trend (73\%); nur 31 Pegel $(27 \%)$ weisen eine Abnahme auf. Bei der Bewertung der Ergebnisse muss berücksichtigt werden, dass die an den Pegeln ermittelten zunehmenden Trends nur zu einem Drittel (33\%) signifikant sind, bei einem relativ niedrig gewählten Signifikanzniveau von $\alpha \geq 80 \%$ (entsprechend einer Irrtumswahrscheinlichkeit $\leq 20 \%$ ). An den Pegeln mit abnehmenden Trends sind lediglich $20 \%$ signifikant.

Das hydrologische Winterhalbjahr zeigt mit zunehmenden Trends an $75 \%$ der Pegel ein dem gesamten hydrologischen Jahr weitgehend ähnliches Verhalten mit signifikant zunehmenden Trends an $41 \%$ aller Pegel. Im hydrologischen Sommerhalbjahr zeigen nur $59 \%$ der Pegel ansteigende Trends der Hochwasserabflüsse, davon ungefähr die Hälfte (47\%) mit signifikanten $\mathrm{Zu}$ nahmen. Bezogen auf die 115 betrachteten Pegel ist im Mittel der Anteil von Pegeln mit signifikant zunehmendem Trend (30\%) höher als von Pegeln mit abnehmendem Trend (5\%).

Bei der Betrachtung der einzelnen Bundesländer treten nur geringfügige Unterschiede im Verhalten der Hochwasserabflüsse für den Zeitraum 1932-2010 auf: Während in Baden-Württemberg bis zu $90 \%$ der Pegel Zunahmen im Gesamtjahr und im Winterhalbjahr zeigen, sind es in Bayern und Rheinland-Pfalz mit 75 bzw. $70 \%$ der Pegel geringfügig weniger. Im Sommerhalbjahr ist die Anzahl der entsprechenden Pegel in allen drei Bundesländern geringer.

\subsubsection{Ergebnisse für den Rhein}

\section{- Analyse der Abflussdaten seit 1927}

Eine durch die globale Erwärmung bedingte Änderung des hydrologischen Regimes eines Flusssystems ist besonders bei durch Schnee geprägten (nivalen) Abflussregimetypen zu erwarten, da hier die zeitliche Verteilung der Abflüsse im Jahresverlauf von der Schneeschmelze (mit-)geprägt wird. Diese Frage ist für den Rhein besonders relevant, da dieser zu den am stärksten genutzten und bewirtschafteten Flüssen der Erde gehört und entlang des
Flusslaufs Wirtschaftsgüter von sehr hohem Wert konzentriert sind. Der Rhein hat im Oberlauf bis etwa Karlsruhe ein nivales Abflussregime und flussabwärts ein gemischtes (nival-pluvial; pluvial: durch Regen geprägt), d. h. komplexes Abflussregime.

- Abb. 10.4 zeigt die 50\%- und 95\%-Quantile der täglichen Abflüsse, gegliedert nach Monaten, am Oberrhein, Pegel Maxau, für die Perioden 1927-1956 und 1967-1996 (Bronstert et al. 2009). Deutlich zu sehen ist bei den Mittelwasserabflüssen (Median, durchgezogene Linien), dass im zweiten Zeitraum die Jahreszeit der hohen Abflusswerte früher beginnt und dass die zugehörigen Höchstwerte 1 Monat früher auftreten, bei in etwa gleichbleibender Größe. Bei Hochwasserbedingungen (95\%-Quantil, gestrichelte Linien) treten die hohen Abflusswerte sogar 2 Monate früher und zudem verstärkt auf. Das heißt, die 95\%-Quantile der Abflüsse im Februar bis Juni wurden höher, die entsprechenden Werte der Monate August bis November dagegen niedriger. Eine solche zeitliche Verschiebung des nivalen hydrologischen Regimes eines Flusses kann deutlich mit der Klimaerwärmung in Zusammenhang gebracht werden, da die erhöhten Frühjahrstemperaturen und die dadurch hervorgerufenen früheren Schneeschmelzereignisse die Verschiebung der Frühsommerhochwasser aus dem Hochgebirge bedingen.

Die Wirkung einer solchen Änderung des Abflussregimes ist zunächst eine schlichte zeitliche Verschiebung. Da sich das Abflussregime des Mittel- und Niederrheins aber aus zwei überlagernden Regimen zusammensetzt, und zwar aus dem alpinen Regime mit Abflussmaxima im Frühsommer und aus dem nival-pluvialen der Mittelgebirge mit Abflussmaxima im Herbst, Winter oder Frühjahr, bedeutet dies, dass sich die Maxima aus beiden Regimen zeitlich annähern. Bei den bekannten hohen Schadenspotenzialen entlang des Rheins - insbesondere am Niederrhein - bedeutete selbst eine nur geringfügige Erhöhung der Auftretenswahrscheinlichkeit eines Extremhochwassers eine beachtliche Zunahme des Hochwasserrisikos.

$\mathrm{Da}$ am Rhein und seinen Nebenflüssen in den vergangenen Jahrzehnten massive flussbauliche Veränderungen vorgenommen wurden, versuchten Vorogushyn und Merz (2013) die beobachteten Trends der Abflüsse am Rhein in einen Zusammenhang mit 
- Abb. 10.4 Quantile derTagesabflüsse am Rhein, gegliedert nach Monaten, Pegel Maxau. Durchgezogen $50 \%$-Quantil (Median), gestrichelt $95 \%$-Quantil, schwarz 1927-1956, rot 1967-1996. (Bürger 2003, zitiert in Bronstert et al. 2009)

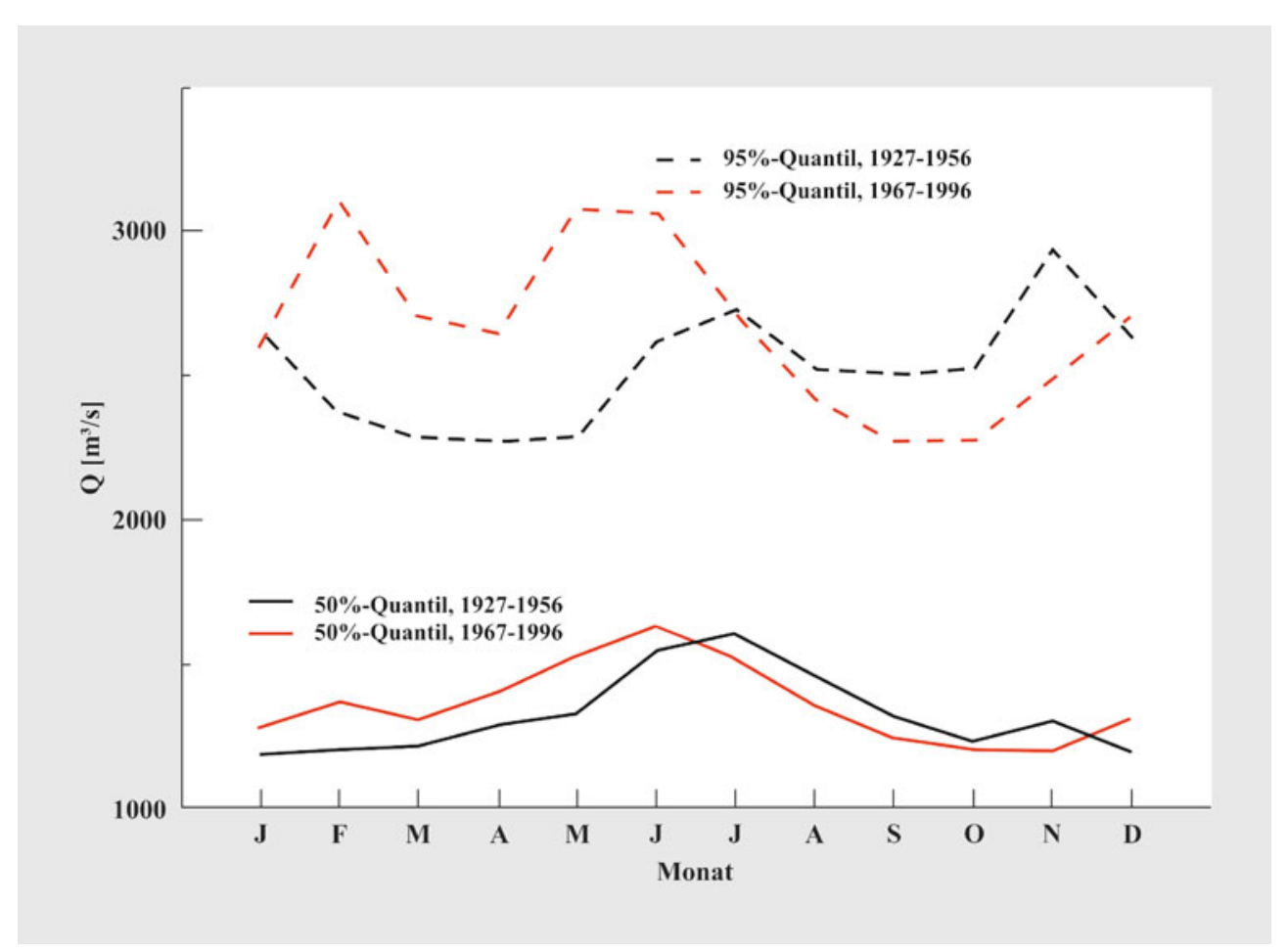

unterschiedlichen Umweltänderungen zu setzen. Sie untersuchten explizit den Einfluss von Flussbaumaßnahmen wie den Bau der Staustufen am Oberrhein mit umfangreichen Verlusten an Überflutungsflächen im Zeitraum 1957-1977 und den Einsatz von Poldern auf die beobachtete Veränderung von Jahresmaximalabflüssen im Zeitraum 1952-2009. Methodisch wurde diese Frage durch eine Homogenisierung der beobachteten Hochwasserzeitreihen am Rhein von Karlsruhe-Maxau bis zur deutsch-niederländischen Grenze angegangen: Es wurden Hochwasserzeitreihen am Rhein für die hypothetische Situation ohne Flussbaumaßnahmen abgeleitet. Anschließend wurden die Trends in den beobachteten und homogenisierten Hochwasserzeitreihen verglichen.

Die Ergebnisse zeigen, dass die homogenisierten Hochwasserzeitreihen nur unwesentlich reduzierte Trends gegenüber den Trends in den beobachteten Zeitreihen aufweisen (bis max. $15 \%$ geringere relative Änderung). Vorogushyn und Merz (2013) schlussfolgern dazu, dass die Flussbaumaßnahmen nur einen geringen Einfluss auf die beobachteten Trends hatten. Ein Großteil der Veränderung sollte somit durch die Summe von Klima- und Landnutzungsänderungen sowie von Einflüssen der Wasserbaumaßnahmen in den Zuflüssen hervorgerufen werden. Dieses Ergebnis stützt somit die Hypothese von Petrow et al. (2009), wonach der Klimaeinfluss die Trends der Hochwasserabflüsse am Rhein dominiert. Diese Aussage gilt allerdings nur mit zwei Einschränkungen: Zum einen wurden die weitreichenden Flussbaumaßnahmen vor dem Zweiten Weltkrieg und im 19. Jahrhundert nicht in diese Analyse einbezogen. Zum anderen wirkt die Bereitstellung zusätzlichen Retentionsvolumens (Retention $=$ Wasserrückhalt) nur bei sehr großen - d. h. seltenen - Ereignissen, also etwa bei einem Wiederkehrintervall von 50 bis 100 Jahren und darüber abflussreduzierend.

Umfangreiche Untersuchungen der Internationalen Hochwasserstudienkommission (HSK 1978) zeigen etwa für den
Oberrhein, Pegel Maxau und Worms deutliche Einflüsse des Stauhaltungsbaus auf die Hochwasserdynamik. Der hier geführte Nachweis der Hochwasserverschärfung im Oberrhein als Folge des Oberrheinausbaus führte zu einer vertraglichen Vereinbarung zwischen Deutschland und Frankreich, die u.a. umfangreiche Retentionsmaßnahmen zur Kompensation der Hochwasserverschärfung durch den Oberrheinausbau vorsieht, von denen bis 2013 rund $60 \%$ des vereinbarten Retentionsvolumens einsatzbereit waren. Auch detaillierte Untersuchungen der Internationalen Kommission zum Schutz des Rheins IKSR (2012) weisen auf den deutlichen Einfluss von Flussbau- und Retentionsmaßnahmen auf die Hochwasserverhältnisse am Rhein hin.

\section{- Modellierungsergebnisse zu künftigen Klimabedingungen}

Im Projekt KLIWA werden auch Simulationen für die Abflussbedingungen im Rhein unter Klimabedingungen für die „nahe Zukunft" von 2021 bis 2050 durchgeführt. Exemplarisch werden hier Ergebnisse bis zum Pegel Worms (Größe des Einzugsgebiets ca. $69.000 \mathrm{~km}^{2}$ ) gezeigt. Zur Simulation der Hydrologie wurde in einer $1-\mathrm{km}^{2}$-Auflösung das Modellsystem LARSIM eingesetzt. Für die Hydrodynamik des Flusslaufs des Oberrheins zwischen Basel und Worms kam das sogenannte synoptische Rheinmodell (Modellinformation s. KLIWA 2013) zum Einsatz. Wie bereits dargelegt, ist zu beachten, dass das Abflussregime des Oberrheins aufgrund der Dominanz der Zuflüsse aus den schweizerischen Alpen nival geprägt ist, mit einem Abflussmaximum im Sommer. Dagegen sind die deutschen Zuflüsse z. B. aus dem Schwarzwald pluvial geprägt, mit einem Abflussmaximum im Winter.

- Tab. 10.2 zeigt die mit dem regionalen Klimamodell CCLM ermittelten Klimaänderungssignale - Temperatur und Niederschlag - bis 2050 für drei CCLM-Realisationen auf Basis des Emissionsszenarios SRES A1B. Es ergibt sich eine Niederschlags- 
- Tab. 10.2 Veränderung von Temperatur und Niederschlag im Rheineinzugsgebiet bei Vergleich der Zukunft (2021-2050) mit dem Ist-Zustand (1971-2000) auf Basis von CCLM 4.8

\begin{tabular}{|l|l|l|l|l|}
\hline $\begin{array}{l}\text { ECHAM 5, A1B, } \\
\text { CCLM 4.8 }\end{array}$ & \multicolumn{2}{|l|}{$\begin{array}{l}\text { Sommerhalbjahr } \\
\text { (Mai-Oktober) }\end{array}$} & $\begin{array}{l}\text { Winterhalbjahr } \\
\text { (November-April) }\end{array}$ \\
\hline & $\begin{array}{l}\text { Tempe- } \\
\text { ratur } \\
\left({ }^{\circ} \mathrm{C}\right)\end{array}$ & $\begin{array}{l}\text { Nieder- } \\
\text { schlag } \\
(\%)\end{array}$ & $\begin{array}{l}\text { Tempe- } \\
\text { ratur } \\
\left({ }^{\circ} \mathrm{C}\right)\end{array}$ & $\begin{array}{l}\text { Nieder- } \\
\text { schlag } \\
(\%)\end{array}$ \\
\hline Realisation 1 & $+1,3$ & $-3,8$ & $+0,9$ & $+7,6$ \\
\hline Realisation 2 & $+1,2$ & $-6,1$ & $+1,3$ & $+11,4$ \\
\hline Realisation 3 & $+0,9$ & $-2,2$ & $+0,9$ & $+3,1$ \\
\hline
\end{tabular}

abnahme im Sommerhalbjahr und eine Zunahme im Winter (KLIWA 2013).

Die Ergebnisse für die mittleren monatlichen Hochwasserabflüsse (MoMHQ) auf Basis der genannten drei CCLMRealisationen für das Szenario „Nahe Zukunft“ (2021-2050), SRES A1B, sind in $\bullet$ Abb. 10.5 für den Pegel Worms dargestellt. Die Unterschiede zwischen den verschiedenen Realisationen von CCLM sind teilweise ausgeprägt. Aus Gründen der besseren Übersichtlichkeit ist als Vergleich anstelle des jeweils simulierten Ist-Zustands der mit meteorologischen Messdaten simulierte Ist-Zustand eingetragen (grüne Linie). Zudem sind auch frühere Ergebnisse auf Basis zweier Varianten des statistischen regionalen Klimamodells WETTREG eingetragen, die größere Abweichungen aufweisen. Beim dem noch eher nival geprägten Abflussregime des Rheinpegels Worms macht sich für die Zukunft auch der Einfluss des Neckars als zusätzliches pluviales Abflussregime bemerkbar. Dies zeigt sich dann an den höheren Abflüssen im Winterhalbjahr. Auf Basis der CCLMKlimaprojektionen wurde im Winterhalbjahr eine Zunahme im Mittel um $8 \%$, im Sommerhalbjahr eine Abnahme um 4\% ermittelt (KLIWA 2013).

Die Abflusszeitreihen bis 2050 wurden extremwertstatistisch ausgewertet und den entsprechenden Ergebnissen des si- mulierten Ist-Zustands gegenübergestellt. In - Abb. 10.6 sind für Hochwasserabflüsse unterschiedlicher Wiederkehrintervalle an verschiedenen Pegeln im Rheineinzugsgebiet die relativen Veränderungen zwischen simulierter Zukunft und simuliertem Ist-Zustand dargestellt (KLIWA 2013). Es ergibt sich meist eine Tendenz zu höheren Abflüssen, d. h., der Faktor auf der y-Achse ist größer als 1: Die Zunahme liegt z. B. beim HQ100 (100-jähriges Hochwasser) bei den Pegeln am Oberrhein mit nivalem Regime bei 3-5\% (Basel, Maxau, Worms) im Bereich der statistischen Unschärfe und fällt somit geringer aus als bei den Pegeln mit pluvialem Regime wie etwa beim Pegel Rockenau/Neckar mit $12 \%$.

\subsubsection{Ergebnisse für das obere Elbegebiet}

Das „obere Elbegebiet“ umfasst hier den Mittelgebirgsteil des Elbegebiets - im Wesentlichen Riesen-, Erz- und Elstergebirge. Wenngleich Dresden damit streng genommen nicht zum Oberlauf des Flusses gehört, schließt die Betrachtung dennoch Hochwasser bis zum Elbepegel Dresden mit ein.

\section{- Analyse der Abflussdaten der letzten 150 Jahre}

Die Jahreshöchstwerte des Durchflusses am Pegel Dresden zeigen über die letzten ca. 150 Jahre einen abnehmenden Trend (Kundzewicz und Menzel 2005; Menzel 2008). Dies könnte auf ein Klimasignal hindeuten. Denn es ist bekannt, dass sich die Häufigkeit starker winterlicher Hochwasser in der Elbe in diesem Zeitraum verringert hat (Mudelsee et al. 2003; Kap. 11) und dies auf eine geringere Bedeutung von Schneeschmelze für die Hochwasserentstehung und einen Rückgang der winterlichen Eisbedeckung und der damit häufig verbundenen Eisstauereignisse durch wärmere Wintertemperaturen zurückgeführt werden kann. Durch Flusslaufverkürzungen und Begradigungen der Elbe hat sich auch die Fließgeschwindigkeit der Elbe erhöht, was die Ausbildung einer winterlichen Eisdecke ebenfalls verzögert. Weiterhin reduzieren Kühlwasser- und Salzeinträge

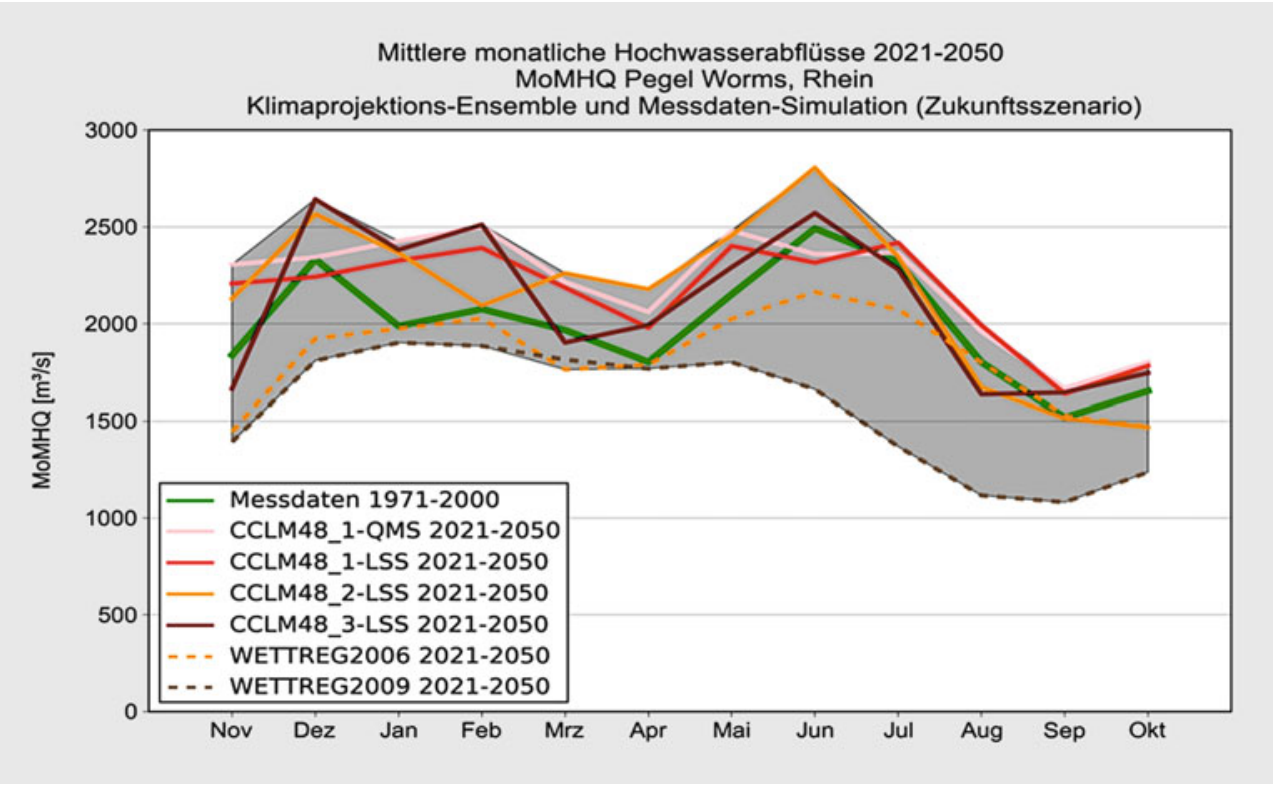

- Abb. 10.5 Mittlere monatliche Hochwasserabflüsse am Pegel Worms/Rhein beim Zukunftsszenario 2021-2050. (KLIWA 2013) 
- Abb. 10.6 Extremwertstatistische Hochwasserauswertungen bis Worms. (KLIWA 2013)

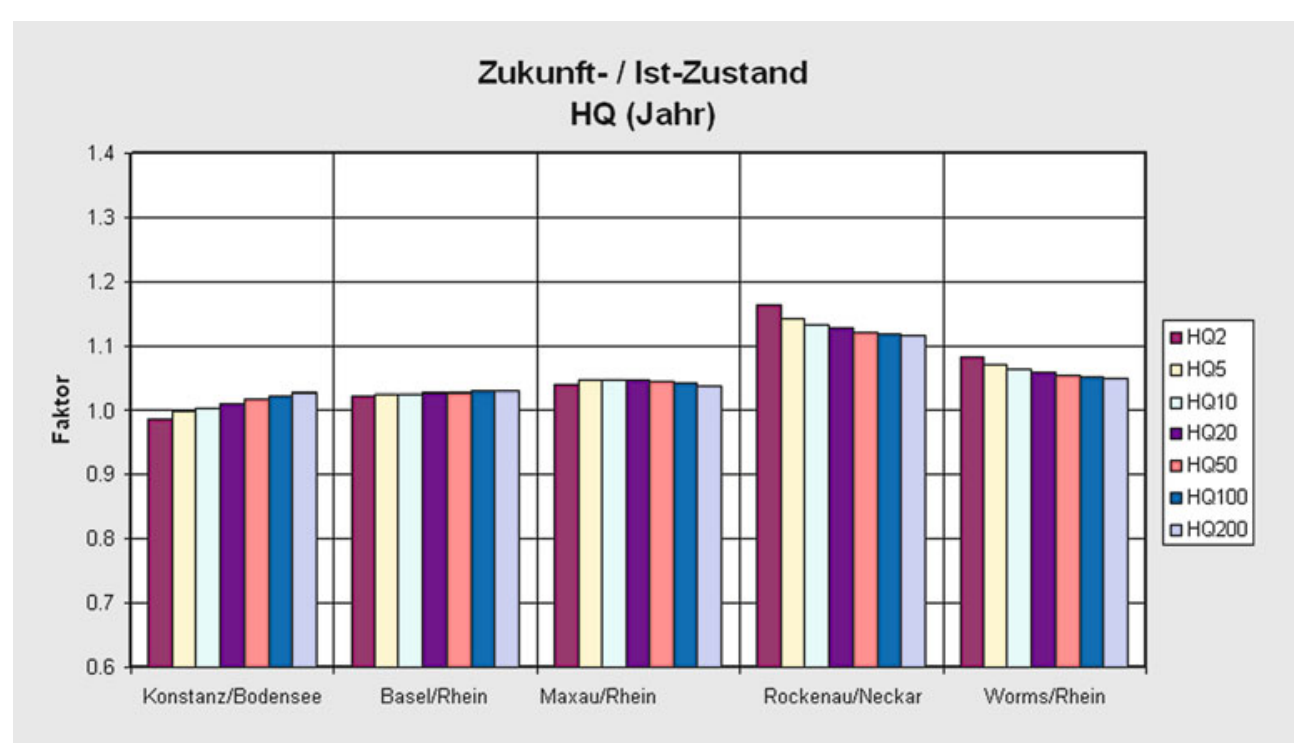

die Eisentstehung. Während das Hochwasser von 1845 im März auftrat, also im Winterhalbjahr, wie der größte Teil der Elbehochwasser in den letzten Jahrhunderten, handelte es sich bei den Hochwasserereignissen 2002 und 2013 um Sommerfluten. Solche extremen Sommerhochwasser kommen vor allem durch großräumige, langanhaltende und ergiebige Niederschläge im Mittelgebirgseinzugsgebiet der Elbe zustande. Diese werden durch advektive Wetterlagen, hier durch den großräumigen Transport warm-feuchter auf relativ kalte aufgleitende Luftmassen, bedingt. Sie werden verstärkt durch orografische Effekte, d. h., der Regen verstärkt sich durch Hebung der Luftmassen an Gebirgen. Wenn z. B. Zugbahnen der sogenannten Vb-Zyklone (eine Wetterlage, die gekennzeichnet ist durch die Zugbahn eines Tiefdruckgebiets von Italien hinweg nordostwärts) (Kundzewicz et al. 2005), die Quellgebiete von Elbe und Oder queren, können solche Konstellationen auftreten. Ihre absolute Zahl ist jedoch so gering, dass sich daraus keine statistisch signifikanten Trends erkennen lassen. Somit ist an der Elbe bis zum Pegel Dresden in den letzten 150 Jahren bislang keine statistisch signifikante Erhöhung der Hochwasserhäufigkeit detektierbar ( Kap. 11).

\section{- Modellierungsergebnisse zu künftigen Klimabedingungen}

Bezüglich der zukünftigen Entwicklung der Abflussverhältnisse im oberen Elbegebiet ist zuerst auf die als sicher geltende $\mathrm{Zu}$ nahme der mittleren Lufttemperaturen hinzuweisen. Temperaturbedingt höhere Regenanteile an den Winterniederschlägen würden sicherlich das zeitliche Auftreten und die Höhe von Abflussspitzen bzw. von Hochwasserereignissen verändern. Die Frühjahrsschmelze findet entweder zeitlich eher oder mangels Schneebedeckung kaum noch statt. Menzel (2008) hat anhand des Einzugsgebiets der Weißen Elster gezeigt, dass sich in einem hydrologischen Szenario (Basis: statistisches downscaling und IPCC SRES A1-Szenario) für den Zeitraum 2021-2050 die mittlere Schneedeckenandauer in diesem Gebiet gegenüber dem Referenzzeitraum 1961-1990 um ein Drittel verkürzt. Einer Erhöhung der winterlichen Abflüsse in den Mittelgebirgsregionen stehen verringerte Abflüsse infolge erhöhter Verdunstungsaktivität in den Sommermonaten gegenüber. Das wird vermutlich $\mathrm{zu}$ einem deutlich ausgeprägter verlaufenden Jahresgang der Abflussregime der Elbe und ihrer Zuflüsse führen. Menzel und Bürger (2002) zeigen für das Einzugsgebiet der Mulde, dass dem gewählten Szenario zufolge (statistisches downscaling auf Basis des IPCC-Szenarios IS95a [ältere Version der SRES-Szenarien]) sowohl die mittleren als auch die mittleren saisonalen Abflüsse zum Teil deutlich zurückgehen, was von Menzel (2008) für das Einzugsgebiet der Weißen Elster bestätigt wurde. Beide Studien beinhalten einen prognostizierten großräumigen Rückgang der Jahresmittel des Niederschlags, was auch von Christensen und Christensen (2003) ähnlich projiziert wird. Diese Aussagen betreffen die mittleren saisonalen Abflussverhältnisse.

Simulationen zum künftigen Auftreten von Starkniederschlägen sind in der für Hochwasserstudien erforderlichen räumlichzeitlichen Auflösung derzeit kaum verfügbar und mit nicht quantifizierbaren Unsicherheiten behaftet (Bronstert et al. 2007), insbesondere für das gebirgige Einzugsgebiet der Elbe. Christensen und Christensen (2003) kommen in ihrer Untersuchung zu dem Ergebnis, dass in weiten Teilen Europas - so auch im oberen Elbeeinzugsgebiet - die zukünftigen Niederschlagsintensitäten in den Sommermonaten deutlich ansteigen könnten, auch wenn die mittleren Sommerniederschlagsmengen abnehmen. $\mathrm{Zu}$ ähnlichen Ergebnissen kommen Kundzewicz et al. (2005) für die Quellgebiete von Elbe, Oder und Weichsel. Sie argumentieren, dass potenziell hochwasserauslösende Vb-Zyklone in Zukunft noch intensivere Niederschläge als bisher liefern würden. Es bleibt allerdings offen, inwieweit diese möglicherweise zunehmenden Niederschlagsintensitäten das Hochwasserrisiko im oberen Elbegebiet verschärfen könnten oder ob ein genereller Trend zur Abnahme mittlerer Niederschlagsmengen die Häufigkeiten und Intensitäten von Hochwasser künftig reduziert. 


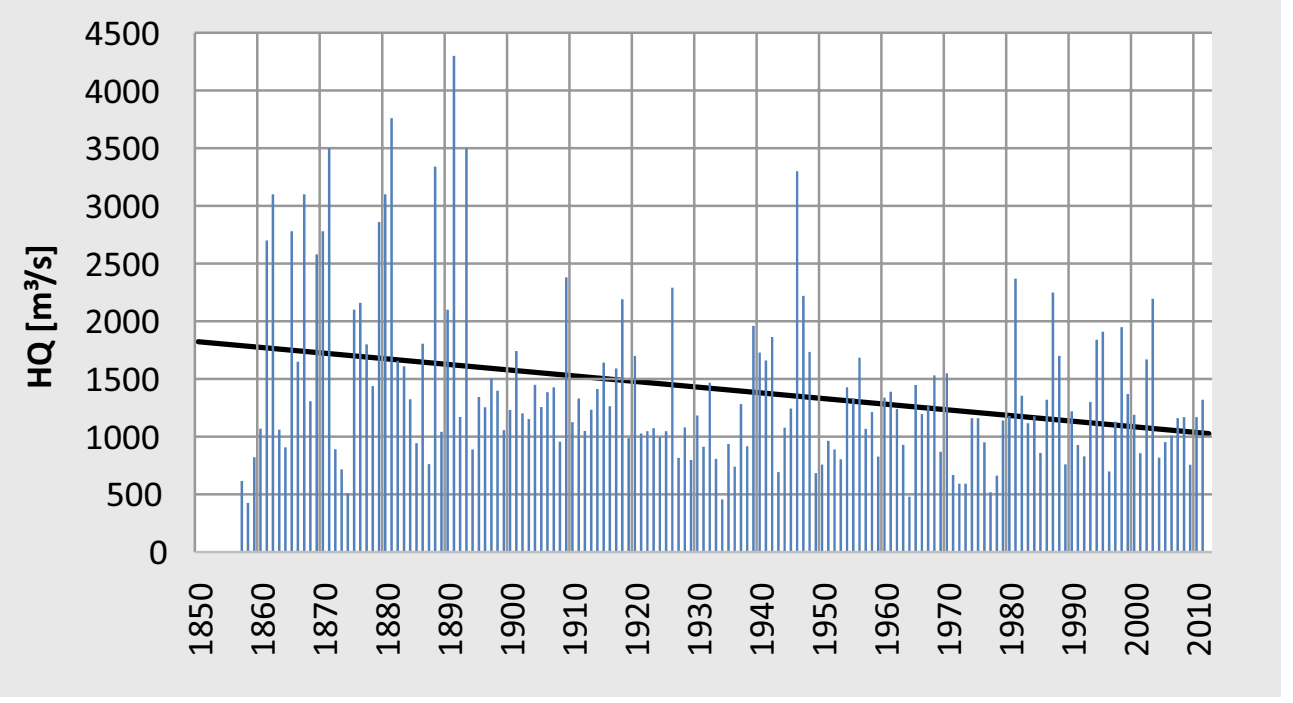

- Abb. 10.7 Jährliche Abflussmaxima am Pegel Intschede (Weser) von 1857-2011 sowie der dazugehörige lineare Trend
10.1.5 Ergebnisse für das Weser- und Emsgebiet

- Analyse der Abflussdaten der letzten 150 Jahre

Analysen der Hochwasserentwicklung an der Weser zeigen, dass die maximalen jährlichen Abflüsse an den Quellflüssen Werra und Fulda zwischen 1950 und 2005 signifikant zugenommen haben (Petrow und Merz 2009; Bormann et al. 2011). Die Weserpegel flussabwärts bis zu den Pegeln Vlotho und Porta weisen für denselben Untersuchungszeitraum ebenfalls signifikant steigende Hochwasserabflüsse auf. Werden Beobachtungen mehrerer Dekaden vor 1950 bei der Trendanalyse berücksichtigt, sind diese Trends aber nur mehr schwach signifikant, wofür hier eine Irrtumswahrscheinlichkeit von $>10 \%$ angesetzt wird. Weiter flussabwärts führen die Zuflüsse aus östlicher Richtung von Aller und Leine zu einer abnehmenden Signifikanz der positiven Trends. Jahreszeitliche Analysen ergaben, dass die Weser durch die $\mathrm{Zu}$ nahme von Winterhochwassern seit Mitte des 20. Jahrhunderts geprägt ist (Petrow und Merz 2009), was auch die Ergebnisse der gesamtjährlichen Analyse dominiert. Sommerliche Hochwasser zeigen für den analysierten Zeitraum keine zunehmende Tendenz.

Die Trends im Abflussverhalten zwischen 1950 und 2005 stehen in einem engen statistischen Zusammenhang mit einem veränderten Niederschlagsverhalten in den jeweiligen Einzugsgebieten (Bormann 2010). Die Winter sind durch zunehmende maximale Niederschläge geprägt, wie Haberlandt et al. (2010) sowohl für 24-Stunden-Niederschläge als auch für 5-Tages-Niederschläge gezeigt haben. Diese Zunahme spiegelt sich in den Trends steigender Hochwasserabflüsse wider (Petrow und Merz 2009; Bormann et al. 2011).

Die Trends der Spitzenabflüsse an der Ems zeigen dieselben Muster wie die an der Weser. Winterhochwasser nahmen von 1951 bis 2002 am Oberlauf zu (Petrow und Merz 2009), während im Sommer kein Trend zu erkennen ist. Insgesamt führte dies zu einer statistisch signifikanten Zunahme der jährlichen Höchstabflüsse am Oberlauf (z. B. Pegel Greven, Bormann et al. 2011). Ähnlich wie am Rhein werden die Spitzenabflüsse allerdings auch von flussbaulichen Veränderungen beeinflusst (Busch et al. 1989;
Bormann et al. 2011), die zum Teil zu einer Kompensation von Abflusstrends, an einigen Pegeln aber auch zu einer Verstärkung des Trends geführt haben (z. B. Pegel Rethem/Aller, Herrenhausen/Leine, Rheine/Ems).

Während die maximalen Hochwasserabflüsse der letzten 50-60 Jahre vielfach steigende Trends aufweisen, zeigen Vergleiche mit Pegelmessungen aus dem 19. Jahrhundert und die Analyse längerer Datenreihen, dass die seit 1950 an der Weser aufgetretenen Hochwasserereignisse moderat im Vergleich zu historischen Hochwassern - vor allem aus der zweiten Hälfte des 19. Jahrhunderts - sind (Sturm et al. 2001; Mudelsee et al. 2006; Bormann et al. 2011, - Abb. 10.7). Zwischen 1870 und 1890 tritt eine Häufung von Hochwasserereignissen auf, die die maximalen Abflüsse des 20. Jahrhunderts deutlich übertreffen. Diese Hochwasser wurden aber u. a. durch Eisstau hervorgerufen, was heute aufgrund des Klimawandels und anthropogener Einflüsse zunehmend unwahrscheinlich ist. Für die Ems liegen an keinem der verfügbaren Pegel entsprechende Datenlängen vor, sodass diese Aussage nicht direkt von der Weser auf die Ems übertragbar ist.

\section{- Modellierungsergebnisse zu künftigen Klimabedingungen}

Dieser Abschnitt gibt eine Zusammenschau zum möglichen (projizierten) Einfluss der Klimaänderung auf die Hochwasserabflüsse im $15.000 \mathrm{~km}^{2}$ großen Aller-Leine-Einzugsgebiet in Niedersachsen. Die Ergebnisse stammen aus dem niedersächsischen Forschungsprojekten KliBiW (NLWKN 2012) und KLIFF (NN 2013).

Als globale klimatische Ausgangsinformationen wurden Ergebnisse des globalen Klimamodells ECHAM5 genommen. Für die hier vorgestellten Untersuchungen wurden darauf basierend zwei dynamische downscaling-Datensätze des regionalen Klimamodells REMO („BfG-Realisierung“ und „UBA-Realisierung“) (Jacob et al. 2008) und drei ausgewählte downscaling-Ergebnisse des statistischen Modells WETTREG 2006 (Spekat et al. 2007) herangezogen. Es wurden jeweils 30-jährige Perioden aus dem Kontrolllauf (1971-2000), der das Klima des späten 20. Jahrhunderts widerspiegelt, und aus dem A1B-Zukunftsszenario („Nahe Zukunft“: 2021-2050, „Ferne Zukunft“: 2071-2100) verwendet. Die hydro- 

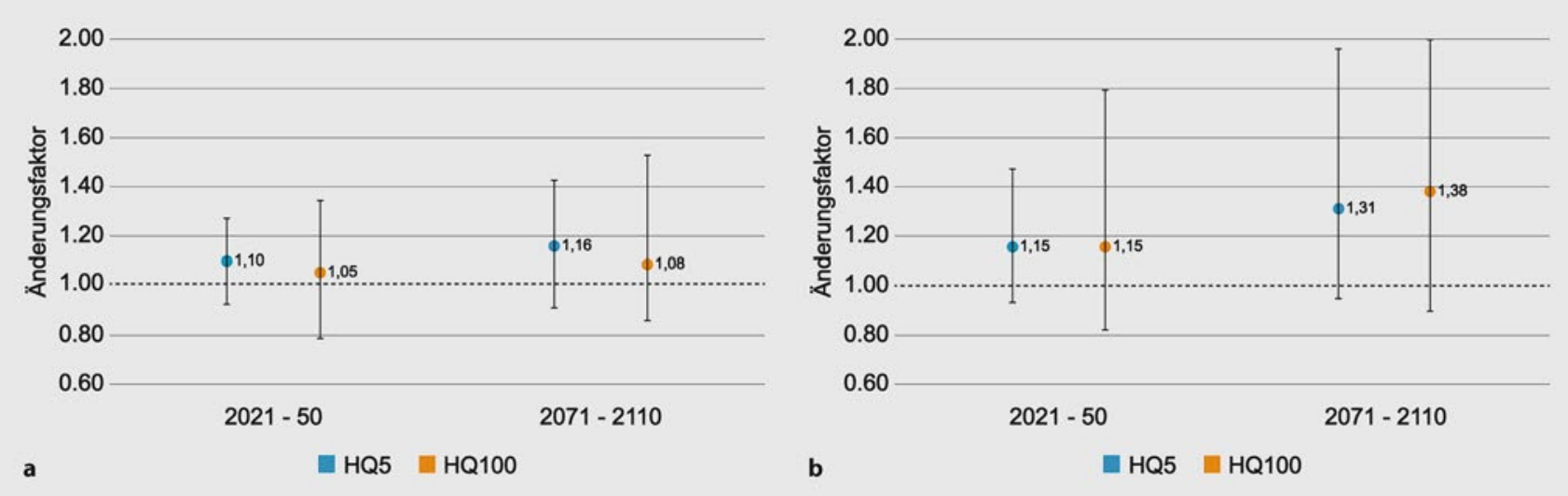

- Abb. 10.8 Projiziertes Änderungssignal für häufige/kleine (HQ5) und seltene/große (HQ100) Hochwasser im Aller-Leine-Flussgebiet. Dargestellt sind die simulierten Änderungsfaktoren für die Perioden 2021-2050 und 2071-2100 gegenüber heutigen Bedingungen. a Mittelwerte für acht relativ große Teilgebiete, b Mittelwerte für sechs kleine Einzugsgebiete.

logischen Simulationen erfolgten mit den Modellen PANTA RHEI (LWI-HYWA 2012) und einer modifizierten Version von HBV (SMHI 2008). Mit PANTA RHEI wurde eine flächendeckende Simulation für das Aller-Leine-Gebiet in Tageszeitschritten durchgeführt. Änderungssignale wurden für acht Referenzpegel mit vergleichsweise großen Einzugsgebieten $\left(800-15.000 \mathrm{~km}^{2}\right)$ analysiert, für die die Modelle validiert werden konnten. Zusätzlich wurden mit PANTA RHEI für sechs ausgewählte, vergleichsweise kleine Teilgebiete $\left(45-600 \mathrm{~km}^{2}\right)$ und mit HBV für 41 Teilgebiete Simulationen in Stundenzeitschritten durchgeführt (Wallner et al. 2013). Die Modelle zeigten für die untersuchten Einzugsgebiete sowohl im Hinblick auf die Wasserbilanz als auch auf die Hochwasserstatistik (z. B. HQ, MHQ) eine gute Wiedergabe (NLWKN 2012) der Beobachtungen im Referenzzeitraum (1971-2000).

- Abb. 10.8 zeigt die simulierten Änderungssignale (relative Änderung zum heutigen Zustand) für die acht relativ großen Einzugsgebiete aus der Tageswertsimulation (PANTA RHEI) und für die sechs kleineren Gebiete aus der Stundenwertsimulation (PANTA RHEI und HBV) für zwei Zukunftszeiträume: für kleine Hochwasser (HQ5), die im statistischen Mittel alle 5 Jahre auftreten, sowie große Hochwasser (HQ100), die im statistischen Mittel einmal in 100 Jahren auftreten. Für die großen Einzugsgebiete werden relativ geringe Zunahmen der Hochwasser projiziert, wobei die HQ5 mit 10 und $16 \%$ Zunahme (d.h. Änderungsfaktor 1,10 bzw. 1,16) prozentual etwas stärker zunehmen als die HQ100 mit 5 und $8 \%$. Die Spannweite der Änderung über alle Realisationen und Einzugsgebiete sind insgesamt sehr groß. Für die kleineren Einzugsgebiete werden etwas stärkere Zunahmen projiziert, wobei hier die Änderung der HQ100 mit 15 und $38 \%$ bedeutender ist als die der HQ5 mit 15 und $31 \%$. Die große Spannweite zeigt jedoch, dass die Unsicherheit von Projektionen für die kleinen Gebiete deutlich höher ist als für die großen.

Die Ergebnisse zeigen eine projizierte Zunahme der Hochwasserabflüsse im Aller-Leine-Gebiet, die physikalisch plausibel ist und mit projizierten Änderungen des Niederschlags korrespondiert. Die Anzahl der hier untersuchten Realisationen (u. a. nur ein globales Klimamodell, ein Klimaszenario) ist jedoch zu gering, um daraus konkrete Anpassungsmaßnahmen ableiten zu können.

\subsection{Sturzfluten und Extremniederschläge kurzer Dauer}

\subsubsection{Spezifika von Sturzfluten}

Sturzfluten sind plötzlich eintretende Hochwasserereignisse, die typischerweise durch kleinräumige, konvektive Starkregenereignisse ausgelöst werden. Sie werden gegenüber Hochwasser in größeren Flüssen durch die Zeit der Verzögerung zwischen dem auslösenden Niederschlagsereignis und dem Eintreten des Hochwasserscheitels abgegrenzt („Reaktionszeit“). Von einer Sturzflut wird typischerweise bei einer Reaktionszeit von nicht mehr als 6 Stunden gesprochen (Borga et al. 2011). Sie treten in Gebieten kleiner als ca. $500 \mathrm{~km}^{2}$ auf, insbesondere in gebirgigen und urbanen Räumen: Dort ist die Aufnahmefähigkeit des Bodens eher gering. Zudem begünstigen geringe Oberflächenrauigkeiten und kleine Gebietsgrößen, zum Teil mit ausgeprägtem Relief, eine rasche Abflusskonzentration. Die besondere Gefährdung, die von Sturzfluten ausgeht, wird durch folgende Merkmale geprägt:

- Geringe Vorwarnzeit: Die Vorwarnzeit ist bei Sturzfluten per definitionem sehr kurz. Die Vorwarnung wird nicht nur durch die rasche Reaktion des Abflusses erschwert, sondern auch durch Probleme bei der Erfassung und Vorhersage der auslösenden Niederschlagsereignisse. Damit sind die Handlungsoptionen zur Einleitung von Gegenmaßnahmen begrenzt. Jonkman (2005) konnte zeigen, dass die Mortalitätsrate, berechnet aus der Zahl der Todesfälle geteilt durch die Zahl der Betroffenen, bei Sturzfluten deutlich größer ist als bei Flusshochwasserereignissen.

- Hohe Fließgeschwindigkeiten: Die für Quelleinzugsgebiete typische hohe Reliefenergie führt zusammen mit extremen Abflüssen nicht nur im Gerinne selbst, sondern auch in Überflutungsbereichen zu sehr hohen Fließgeschwindigkeiten. Zusammen mit der großen Menge und Geschwindigkeit des mitgeführten Materials führt dies potenziell zu extremen Schäden an Gebäuden und der Infrastruktur. 

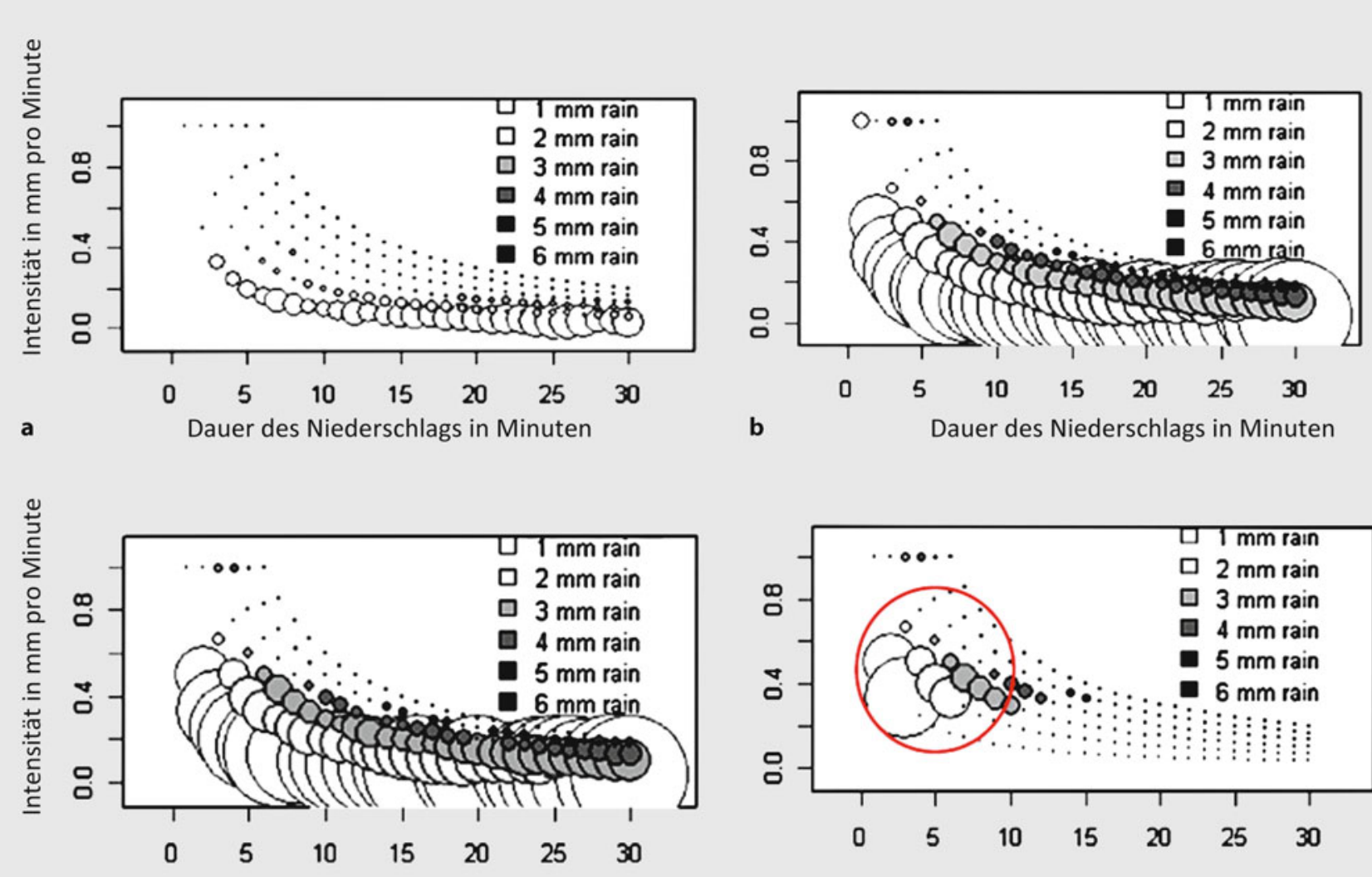

c

Dauer des Niederschlags in Minuten

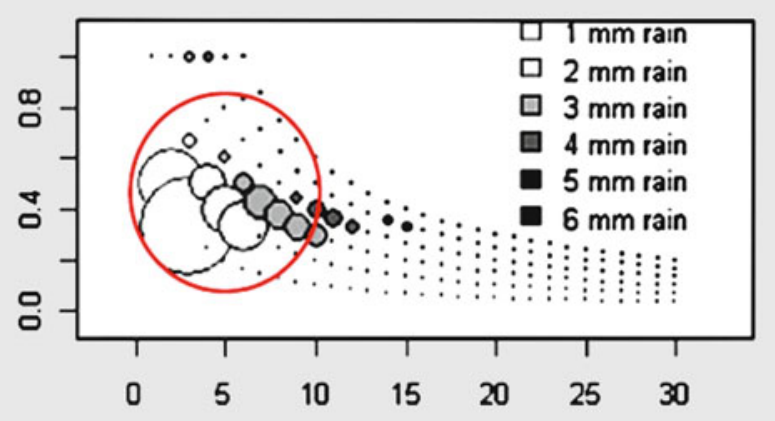

d

Dauer des Niederschlags in Minuten

- Abb. 10.9 Blasendiagramm für die Station Essen: a Positive Trends für Ereignisse mit gegebener Dauer, Intensität und Niederschlagsmenge für die Periode 1940-2009; b wie zuvor, aber für die Periode 1975-2009; c wie zuvor, aber nur mit den signifikanten Trends; d wie c, aber nur mit erosionsrelevanten Ereignissen. Die Größe der Blasen ist gleich skaliert. Die größten Blasen (in Plot b) entsprechen einem positiven Trend von 0,5 Ereignissen/Jahr, und ein Punkt entspricht einem Null-Trend. (Aus Müller und Pfister 2011)

- Singuläres (chaotisches) Verhalten: Das Ausuferungsund Überflutungsverhalten ist bei Sturzfluten schwer vorhersagbar und wird oft durch singuläre Gegebenheiten maßgeblich beeinflusst. Ein typisches Beispiel dafür sind Verschlüsse von Fließgewässern durch Treibgut an Brücken oder sonstigen Verengungen, die je nach Menge und Beschaffenheit des mitgeführten Materials zu spontanem Rückstau und Änderungen des Fließweges führen können. Spontane Wiederauflösungen derartiger Hindernisse können darüber hinaus zu einer massiven Verstärkung der Abflussspitzen führen.

Daher ist es viel schwieriger, Sturzfluten zu erfassen als Flussüberschwemmungen. Insofern liegen nur wenige fundierte Aussagen zu zeitlichen Veränderungen der Sturzflutgefährdung vor, und es ist nicht möglich, eine regionale Differenzierung wie bei den Flussüberschwemmungen vorzunehmen.

\subsubsection{Datenanalyse zur Entwicklung von hochintensiven Starkregenereignissen}

In einer detaillierten Studie berichten Müller und Pfister (2011) über die Analyse langer Niederschlagszeitreihen, die für acht
Stationen im Emscher-Lippe-Gebiet in Nordrhein-Westfalen in einer außergewöhnlich hohen zeitlichen Auflösung (1 min) für die letzten 70 Jahre (1940er-2009) zur Verfügung standen. Aus diesen Datenreihen wurden Ereignisse mit Dauern von $1 \mathrm{~min}$ bis $30 \mathrm{~min}$ herausgefiltert, die jeweils Niederschlagsmengen von $1-10 \mathrm{~mm}$ überschritten. Ereignisse über einem Schwellenwert der Niederschlagsintensität von $0,3 \mathrm{~mm} / \mathrm{min}$ bzw. $>20 \mathrm{~mm} / \mathrm{h}$ wurden hinsichtlich Trends und Änderungen statistisch untersucht. Die Ergebnisse zeigen, dass für alle untersuchten Stationen die Anzahl dieser kurz andauernden Niederschlagsereignisse mit starken Intensitäten in den letzten Jahrzehnten zugenommen hat. Diese Trends haben sich in den letzten 35 Jahren noch ausgeprägter gezeigt als in der Zeit davor. Die Trendzunahme war besonders in den Sommermonaten von Juli bis September stark ausgeprägt. Diese hoch intensiven Starkregen treten zwischen 4- und 15-mal im Jahr auf. Die festgestellte Zunahme von bis zu 0,5 Ereignissen pro Jahr würde also eine Vervielfachung der Häufigkeit dieser Ereignisse in nur wenigen Jahrzehnten bedeuten. Diese Studie belegt zum ersten Mal quantitativ, dass sich das Auftreten solcher hoch intensiven Regenereignisse im Untersuchungsgebiet deutlich verstärkt hat. Müller und Pfister (2011) diskutieren ihre Ergebnisse bezüglich der Wirkung dieser Änderung des Intensitätsregimes für Erosion auslösende Regenfälle ( $\left.\mathrm{I}_{\text {Regen }}>0,3 \mathrm{~mm} / \mathrm{min}\right)$. In $\bullet$ Abb. 10.9 
sind diese Ergebnisse verschiedenen Zeitperioden, Signifikanzen und Intensitätsschwellenwerte gegenübergestellt. Hier kann ein kritischer Bereich für Erosionsereignisse identifiziert werden (für einen Wertebereich der Niederschlagsintensitäten $I_{\text {Regen }}$ $=0,3-0,7 \mathrm{~mm} / \mathrm{min}$, Niederschlagsdauer $t_{\text {Regen }}=2-10 \mathrm{~min}$ und Niederschlagsmenge $h_{\text {Regen }}=1-3 \mathrm{~mm}$ ), der mit dem roten Kreis gekennzeichnet ist.

Zwei weitere Studien analysierten denselben Datensatz: Die Studie von Fiener et al. (2013) für das gleiche Untersuchungsgebiet bestätigt die Kernaussagen, dass der erosionsrelevante Starkregen seit Mitte der 1970er-Jahre signifikant zunimmt; in ihrer Studie gehen sie von einer Zunahme von $21 \%$ pro Jahrzehnt aus. In der Analyse von 5-Minuten-Dauerstufen der ExUS-Studie (NRW 2010) wurden keine statistisch signifikanten Trends für das Auftreten von Extremereignissen in ihrem Analysezeitraum 1950-2008 gefunden; eine nach Zeiträumen differenzierte Analyse erfolgte in dieser Studie nicht.

Es ist nicht davon auszugehen, dass der Trendanstieg des Betrachtungszeitraums 1975-2009 auf die Verbesserung der Messtechnik (Umstellung von analogen auf digitale Systeme) zurückzuführen ist, da man im Zeitreihenverlauf ab den 1990er-Jahren eher einen kontinuierlichen und nicht einen abrupten Anstieg ausmachen kann. Für die Stadtentwässerung kann der Anstieg dieser Starkregen von Bedeutung sein. In der bisherigen Kanalbemessung wird typischerweise eine Dauerstufe von 15 min für den Konzentrationszeitraum von Abflussspitzen eingesetzt. Es bleibt zu überprüfen, inwiefern Starkregen von geringerer Dauerstufe, aber dafür sehr starken Intensitäten in Zukunft berücksichtigt werden müssen. Für landwirtschaftlich genutzte Flächen könnte ein vermehrtes Auftreten an erosionsrelevanten Starkregen zu einem Anstieg der Bodenerosionserscheinungen an Hängen, Auslaugen der Böden, verstärktem Ausspülen von Nähr- und Schadstoffen und einer Verlagerung dieser Stoffe in die Oberflächengewässer mit entsprechend negativen Auswirkungen auf die Gewässerökologie führen. Die Allgemeingültigkeit dieser Aussage sollte in einem nächsten Analyseschritt für weitere hoch aufgelöste Niederschlagszeitreihen für die Großregion Deutschland/ Zentraleuropa überprüft werden. Dies wird jedoch schwierig sein, da Zeitreihen mit einer vergleichbaren zeitlichen Auflösung und Länge im Normalfall nicht existieren. Des Weiteren steht eine Untersuchung aus, inwieweit die Entstehungsmechanismen von Niederschlägen und ggf. dazugehörigen Großwetterlagen, die Starkregen der beschriebenen Intensitäts- und Dauerstufen auslösen, durch eine mögliche weitere Klimaerwärmung beeinflusst werden.

\subsubsection{Zur künftigen Entwicklung von hoch intensiven Starkregenereignissen}

Um zukünftige Auswirkungen des Klimawandels auf die Häufigkeit und Amplitude von Sturzflutereignissen zu ermitteln, bedürfte es Niederschlagsprojektionen für kurze Dauerstufen kleiner als einer Stunde. Derartige Projektionen sind auf Grundlage gegenwärtiger Simulationsmodelle nicht verfügbar. So betrachtet beispielsweise eine aktuelle Auswertung im Rahmen einer ressortübergreifenden Behördenallianz (DWD 2012) lediglich ex- treme Niederschläge auf Tagesbasis. Alternativ zur unmittelbaren Betrachtung simulierter Niederschlagshöhen aus Klimamodellen hat sich in den vergangen Jahren eine neue Perspektive entwickelt: die Betrachtung der Abhängigkeit extremer Niederschläge kurzer Dauer von der Lufttemperatur. Grundsätzlich hängt der Einfluss der Lufttemperatur auf den Niederschlag stark von der betrachteten zeitlich-räumlichen Skala ab. Der globale Gesamtniederschlag nimmt im Mittel um etwa $3 \%$ pro Grad Erwärmung zu und ist im Wesentlichen über den latenten Wärmefluss, also in erster Linie Verdunstung und Kondensation, beschränkt (Allen und Ingram 2002). Der Zusammenhang zwischen extremen lokalen Niederschlägen und der Lufttemperatur scheint hingegen deutlich stärker ausgeprägt zu sein. Aus langjährigen Beobachtungsreihen in Westeuropa (Lenderink und van Meijgaard 2008), Deutschland (u. a. Haerter und Berg 2009; Haerter et al. 2010; Bürger et al. 2014) und anderen Kontinenten (Panthou et al. 2014 für Kanada, Mishra et al. 2012 für die USA, Hardwick Jones et al. 2010 für Australien) ergaben sich für Extremintensitäten des stündlichen Niederschlags Werte, die recht gut durch die Clausius-Clapeyron-Beziehung beschrieben werden. Diese besagt - verkürzt - nichts anderes, als dass die besonders extremen Kurzzeitereignisse durch nichts limitiert sind als den maximalen Feuchtigkeitsgehalt der Atmosphäre, der seinerseits exponentiell von der Temperatur abhängt. Wahrscheinlich variiert der Zusammenhang zwischen Temperatur und Extremniederschlag auch auf subtäglicher Skala (Loriaux et al. 2013) und ist ferner abhängig von der Wetterlage und von den regionalen hydroklimatischen Bedingungen.

Nichtsdestotrotz bietet sich hiermit eine neue Perspektive, aus Projektionen über die zukünftige Erwärmung auch Veränderungen zukünftiger Niederschlagsextreme kurzer Dauerstufen abzuleiten. So werden gegenwärtig Ansätze entwickelt, die genannten Beziehungen direkt auf globale Klimaprojektionen anzuwenden und Abschätzungen für zukünftiges Kurzfristverhalten zu gewinnen (Bürger et al. 2014).

\subsection{Kurz gesagt}

Flusshochwasser werden in lokale/plötzliche Sturzfluten und in Hochwasser in/an größeren Flüssen unterschieden. Ansätze zur Beurteilung der Klimaänderungswirkungen auf Hochwasser sind die Analyse von Hochwasserzeitreihen und/oder die prozessbasiert-gekoppelte Modellierung. Die erste Methode wurde für die Analyse der in der Vergangenheit beobachteten Bedingungen eingesetzt, die zweite für die Abschätzung der bis ca. $2100 \mathrm{zu}$ erwartenden Entwicklung.

Für Deutschland zeigen sich an größeren Flüssen Trends in den jährlichen Höchstabflüssen für 1951-2002 an ca. 30\% der untersuchten Pegel. Die große Mehrheit dieser Trends ist positiv (also zunehmende Hochwasserwerte). Für verschiedene Hochwasserindikatoren und Flusseinzugsgebiete ergeben sich erhebliche Unterschiede. Die Einzugsgebiete der Donau und des Rheins zeigen die meisten Trends, Weser und Elbe deutlich weniger.

Bezüglich der für Sturzfluten relevanten extremen Niederschlagsintensitäten in kurzen Zeiträumen (wenige Minuten) zeigt eine neue Analyse im Emscher-Lippe-Gebiet, dass dort solche 
Ereignisse in den letzten Dekaden sehr signifikant zugenommen haben, was für agrar- und urbanhydrologische Fragestellungen von hoher Bedeutung sein kann.

Bei den Simulationen der bis ca. 2100 zu erwartenden Hochwasserbedingungen fällt die enorme Unsicherheit der Ergebnisse ins Gewicht. Es wird an manchen Flüssen eine Zunahme der Hochwasserabflüsse projiziert, die bei Pegeln mit nivalem $\mathrm{Re}$ gime geringer ausfällt als bei den Pegeln mit pluvialem Regime. Diese Projektionen sind physikalisch plausibel und korrespondieren mit den projizierten Niederschlagsänderungen. Die Unsicherheiten sind allerdings zu hoch, um aus diesen Ergebnissen bereits quantitativ fundierte Anpassungsmaßnahmen ableiten zu können. Gleichwohl sollten aufgrund der überwiegend positiven Tendenzen bereits jetzt Möglichkeiten der qualitativen Anpassungsmaßnahmen bei neuen Hochwasserschutzmaßnahmen erwogen werden.

\section{Literatur}

Allen MR, Ingram WI (2002) Constraints on future changes in climate and the hydrologic cycle. Nature 419(6903):224-232

Borga M, Anagnostou EN, Bloeschl G, Creutin J-D (2011) Flash flood forecasting, warning and risk management: the HYDRATE project. Environ Sci Policy 14:834-844

Bormann H (2010) Runoff regime changes in German rivers due to climate change. Erdkunde 64(3):257-279

Bormann H, Pinter N, Elfert S (2011) Hydrological signatures of flood trends on German rivers: flood frequencies, flood heights and specific stages. J Hydrol 404:50-66

Bronstert A, Kolokotronis V, Schwandt D, Straub H (2007) Comparison and evaluation of regional climate scenarios for hydrological impact analysis: general scheme and application example. Int J Climatol 27:1579-1594

Bronstert A, Kneis D, Bogena H (2009) Interaktionen und Rückkopplungen beim hydrologischen Wandel: Relevanz und Möglichkeiten der Modellierung. Hydrol Wasserbewirtsch 53(5):289-304

Bürger G (2003) Rhein-Hochwasser und ihre mögliche Intensivierung unter globaler Erwärmung: die Überlagerung von Schmelz- und Niederschlagseffekten. Universität Potsdam, Institut für Geoökologie (unveröffentlichte Studie)

Bürger G, Heistermann M, Bronstert A (2014) Towards sub-daily rainfall disaggregation via Clausius-Clapeyron. J Hydrometeorol 15:1303-1311

Busch D, Schirmer M, Schuchardt B, Ullrich P (1989) Historical changes of the River Weser. Petts GE (Hrsg) Historical change of large alluvial rivers: Western Europe. Wiley, Chichester, S 297-321

Christensen $\mathrm{JH}$, Christensen OB (2003) Severe summertime flooding in Europe. Nature 421:805-806

DWD (2012) Auswertung regionaler Klimaprojektionen für Deutschland hinsichtlich der Änderung des Extremverhaltens von Temperatur, Niederschlag und Windgeschwindigkeit. Abschlussbericht, Oktober 2012, Offenbach, Main, $153 \mathrm{~S}$

Fiener P, Neuhaus P, Botschek J (2013) Long-term trends in rainfall erosivity - analysis of high resolution precipitation time series (1937-2007) from Western Germany. Agric For Meteorol 171-172(2013):115-123

Haberlandt U, Belli A, Hölscher J (2010) Trends in beobachteten Zeitreihen von Temperatur und Niederschlag in Niedersachsen. Hydrol Wasserbewirtsch 54:28-36

Haerter JO, Berg P (2009) Unexpected rise in extreme precipitation caused by a shift in rain type? Nat Geosci 2:372-373

Haerter JO, Berg P, Hagemann S (2010) Heavy rain intensity distributions on varying time scales and at different temperatures. J Geophys Res: Atmospheres 115(D17):2156-2202
Hardwick Jones R, Westra S, Sharma A (2010) Observed relationships between extreme sub-daily precipitation, surface temperature, and relative humidity. Geophys Res Lett 37:L22805

Hartmann DL, Klein Tank AMG, Rusticucci M, Alexander LV, Brönnimann S, Charabi Y, Dentener FJ, Dlugokencky EJ, Easterling DR, Kaplan A, Soden BJ, Thorne PW, Wild M, Zhai PM (2013) Observations: Atmosphere and surface. In: Stocker TF, Qin D, Plattner G-K, Tignor M, Allen SK, Boschung J, Nauels A, Xia Y, Bex V, Midgley PM (Hrsg) Climate change 2013: The physical science basis. Contribution of working group I to the fifth assessment report of the Intergovernmental Panel on Climate Change. Cambridge University Press, Cambridge

Hattermann FF, Kundzewicz ZW, Huang S, Vetter T, Kron W, Burghoff O, Merz B, Bronstert A, Krysanova V, Gerstengarbe F-W, Werner P, HaufY (2012) Flood risk in holistic perspective - observed changes in Germany. In: Kundzewicz ZW (Hrsg) Changes in flood risk in Europe. Special Publication No 10. IAHS Press, Wallingford, Oxfordshire, UK (Ch 11)

Hattermann FF, Kundzewicz ZW, Huang S, Vetter T, Gerstengarbe FW, Werner P (2013) Climatological drivers of changes in flood hazard in Germany. Acta Geophys 61(2):463-477

HSK (1978) Schlussbericht der Hochwasser-Studienkommission für den Rhein Hundecha Y, Merz B (2012) Exploring the relationship between changes in climate and floods using a model-based analysis. Water Resour Res 48:W04512. doi:10.1029/2011WR010527

IKSR (2012) Nachweis der Wirksamkeit von Maßnahmen zur Minderung der Hochwasserstände im Rhein. Internationale Kommission zum Schutze des Rheins, Bericht 199. www.iksr.org/uploads/media/199_d.pdf

Jacob D, Göttel H, Kotlarski S, Lorenz P, Sieck K (2008) Klimaauswirkungen und Anpassung in Deutschland - Phase 1: Erstellung regionaler Klimaszenarien für Deutschland. Climate Change 11/08. Umweltbundesamt, DessauRoßlau

Jiménez Cisneros BE, Oki T, Arnell NW, Benito G, Cogley JG, Döll P, Jiang T, Mwakalil SS (2014) Freshwater resources. In: Field CB, Barros VR, Dokken DJ, Mach KJ, Mastrandrea MD, Bilir TE, Chatterjee M, Ebi KL, Estrada YO, Genova RC, Girma B, Kissel ES, Levy AN, MacCracken S, Mastrandrea PR, White LL (Hrsg) Climate change 2014: Impacts, adaptation, and vulnerability. Part A: Global and sectoral aspects. Contribution of working group II to the fifth assessment report of the Intergovernmental Panel on Climate Change. Cambridge University Press, Cambridge, S 229-269

Jonkman SN (2005) Global Perspectives on Loss of Human Life Caused by Floods. Natural Hazards 34:2

KLIWA (2011) Klimawandel in Süddeutschland - Veränderungen von meteorologischen und hydrologischen Kenngrößen, Klimamonitoring im Rahmen des Kooperationsvorhabens KLIWA, Monitoringbericht 2011

KLIWA (2013) Klimaveränderung und Konsequenzen für die Wasserwirtschaft. Fachvorträge beim 5. KLIWA-Symposium, Würzburg, 06. und 07.12.2012. KLIWA Heft, Bd. 19.

Krysanova V, Müller-Wohlfeil DI, Becker A (1998) Development and test of a spatially distributed hydrological / water quality model for mesoscale watersheds. Ecol Model 106:261-289

Kundzewicz ZW, Menzel L (2005) Natural flood reduction strategies - a challenge. Int J River Basin Management 3(2):125-131

Kundzewicz ZW, Ulbrich U, Brücher T, Graczyk D, Krüger A, Leckebusch G, Menzel L, Pinskwar I, Radziejewski M, Szwed M (2005) Summer floods in Central Europe - climate change track? Nat Hazards 36:165-189

Lenderink G, van Meijgaard E (2008) Increase in hourly precipitation extremes beyond expectations from temperature changes. Nat Geosci 1(8):511-514

Loriaux JM, Lenderink G, De Roode SR, Siebesma AP (2013) Understanding convective extreme precipitation scaling using observations and an entraining plume model. J Atmo Sci 130814132040006. doi:10.1175/JAS-D-12-0317.1

LWI-HYWA (2012) PANTA RHEI Benutzerhandbuch - Programmdokumentation zur hydrologischen Modellsoftware. Leichtweiß-Institut für Wasserbau (LWI), Abteilung Hydrologie, Wasserwirtschaft und Gewässerschutz (HYWA), Technische Universität Braunschweig, Braunschweig

Menzel L (2008) Modellierung hydrologischer Auswirkungen von Klimaänderungen. In: Kleeberg H-B (Hrsg) Klimawandel - Was kann die Wasserwirtschaft tun? Forum für Hydrologie und Wasserbewirtschaftung, Bd. 24.08. Eigenverlag, Hennef, S 35-51 
Menzel L, Bürger G (2002) Climate change scenarios and runoff response in the Mulde catchment (Southern Elbe, Germany). J Hydrol 267:53-64

Merz B, Vorogushyn S, Uhlemann S, Delgado J, Hundecha Y (2012) HESS Opinions, More efforts and scientific rigour are needed to attribute trends in flood time series. Hydrol Earth Syst Sci 16:1379-1387. doi:10.5194/hess16-1379-2012

Mishra V, Wallace JM, Lettenmaier DP (2012) Relationship between hourly extreme precipitation and local air temperature in the United States. Geophys Res Lett 39:L16403

Mudelsee M, Börngen M, Tetzlaff G, Grünewald G (2003) No upward trends in the occurrence of extreme floods in central Europe. Nature 425:166-169

Mudelsee M, Deutsch M, Börngen M, Tetzlaff G (2006) Trends in flood risk of the river Werra (Germany) over the past 500 years. Hydrol Sci J 51(5):818-833

Müller EN, Pfister A (2011) Increasing occurrence of high-intensity rainstorm events relevant for the generation of soil erosion in a temperate lowland region in Central Europe. J Hydrol 411(3):266-278

NLWKN (2012) Globaler Klimawandel - Wasserwirtschaftliche Folgenabschätzung für das Binnenland. Oberirdische Gewässer, Bd. 33. Niedersächsischer Landesbetrieb für Wasserwirtschaft, Küsten- und Naturschutz, Hildesheim

NN (2013) KLIFF - Klimafolgenforschung Niedersachsen. http://www.kliff-niedersachsen.de.vweb5-test.gwdg.de/

NRW (2010) ExUS Extremwertstatistische Untersuchungen von Starkregen in Nordrhein-Westfalen, aqua_plan $\mathrm{GmbH}$, hydro \& meteo $\mathrm{GmbH}$ \& Co. KG und dr. papadakis $\mathrm{GmbH}$

Ott I, Duethmann D, Liebert J, Berg P, Feldmann H, Ihringer J, Kunstmann $\mathrm{H}$, Merz B, Schaedler G, Wagner S (2013) High-resolution climate change impact analysis on medium-sized river catchments in Germany: An ensemble assessment. J Hydrometeorol 14:1175-1193. doi:10.1175/JHM-D-12-091.1

Panthou G, Mailhot A, Laurence E, Talbot G (2014) Relationship between surface temperature and extreme rainfalls: A multi-time-scale and event-based analysis. J Hydrometeorol 15:1999-2011

Petrow T, Merz B (2009) Trends in flood magnitude, frequency and seasonality in Germany in the period 1951 - 2002. J Hydrol 371(1-4):129-141

Petrow T, Zimmer J, Merz B (2009) Changes in the flood hazard in Germany through changing frequency and persistence of circulation patterns. Nat Hazards Earth Syst Sci (HNESS) 9:1409-1423 (www.nat-hazards-earth-systsci.net $/ 9 / 1409 / 2009)$

Schmocker-Fackel P, Naef F (2010) More frequent flooding? Changes in flood frequency in Switzerland since 1850. J Hydrol 381:1-2 (1-8)

SMHI (2008) Integrated hydrological modelling system - Manual version 6.0. Swedish Meteorological and Hydrological Institute

Spekat A, Enke W, Kreienkamp F (2007) Neuentwicklung von regional hoch aufgelösten Wetterlagen für Deutschland und Bereitstellung regionaler Klimaszenarios auf der Basis von globalen Klimasimulationen mit dem Regionalisierungsmodell WETTREG auf der Basis von globalen Klimasimulationen mit ECHAM5/MPI-OM T63L31 2010 bis 2100 für die SRESSzenarios B1, A1B und A2, Forschungsprojekt im Auftrag des Umweltbundesamtes (UBA), FuE-Vorhaben Förderkennzeichen 20441138

Sturm K, Glaser R, Jacobeit J, Deutsch M, Brazdil R, Pfister C, Luterbacher J, Wanner $\mathrm{H}$ (2001) Hochwasser in Mitteleuropa seit 1500 und ihre Beziehung zur atmosphärischen Zirkulation. Petermanns Geographische Mitteilungen 145(6):14-23

Vorogushyn S, Merz B (2013) Flood trends along the Rhine: the role of river training. Hydrol Earth Syst Sci 17(10):3871-3884

Wallner M, Haberlandt U, Dietrich J (2013) A one-step similarity approach for the regionalization of hydrological model parameters based on Self-Organi zing Maps. J Hydrol 494:59-71
Open Access Dieses Kapitel wird unter der Creative Commons Namensnennung 4.0 International Lizenz (http://creativecommons.org/ licenses/by/4.0/deed.de) veröffentlicht, welche die Nutzung, Vervielfältigung, Bearbeitung, Verbreitung und Wiedergabe in jeglichem Medium und Format erlaubt, sofern Sie den/die ursprünglichen Autor(en) und die Quelle ordnungsgemäß nennen, einen Link zur Creative Commons Lizenz beifügen und angeben, ob Änderungen vorgenommen wurden.

Etwaige Abbildungen oder sonstiges Drittmaterial unterliegen ebenfalls der genannten Creative Commons Lizenz, sofern sich aus der Abbildungslegende oder der Quellreferenz nichts anderes ergibt. Sofern solches Drittmaterial nicht unter der genannten Creative Commons Lizenz steht, ist eine Vervielfältigung, Bearbeitung oder öffentliche Wiedergabe nur mit vorheriger Zustimmung des betreffenden Rechteinhabers oder auf der Grundlage einschlägiger gesetzlicher Erlaubnisvorschriften zulässig. 